\title{
MEASURING IMPEDANCE OF TISSUES USING A MICROFABRICATED MICROELECTRODE ARRAY
}

\author{
A Thesis Presented to the Faculty \\ of the College of Engineering \\ California Polytechnic University \\ San Luis Obispo
}

\author{
In Partial Fulfillment of the Requirements \\ for the Degree of \\ Master of Science in Biomedical Engineering
}

By

Ashwini Bhat

December 2012 
(C)2012

Ashwini Bhat

ALL RIGHTS RESERVED 


\section{COMMITTEE MEMBERSHIP}

TITLE:

AUTHOR:

DATE SUBMITTED:

COMMITTEE CHAIR:

COMMITTEE MEMBER:

COMMITTEE MEMBER:
Measuring Impedance Of Tissues Using A

Microfabricated Microelectrode Array

Ashwini Bhat

December 2012

Dr. Lily Laiho, Associate Professor

Dr. Richard Savage, Department Chair of Materials Engineering

Dr. Robert Szlavik, Associate Professor 


\section{ABSTRACT \\ MEASURING IMPEDANCE OF TISSUES USING A MICROFABRICATED MICROELECTRODE ARRAY}

By

Ashwini Bhat

This thesis looks at the possibility of using impedance spectroscopy for differentiating tissue, using a microelectrode array (MEA). The thesis first discusses the background and the motivation for this thesis. It covers the certain basic concepts of the human skin starting from the top epidermis layer all the way to the deep dermis layers of the skin. Then it discusses different types of skin cancer and how they occur, in humans. It also discusses various microfabrication techniques such as oxidation, wet etching, sputtering and photolithography for the creation of a MEA in order to test the tissue. The microfabricated MEA is then used to measure impedance across cooked and raw chicken at different frequencies in order to see if the two types of tissues can be differentiated using their respective impedances. The data shows that the MEA was not able to successfully differentiate the two types of the tissues. It does however list multiple improvements in the fabrication of the MEA and improvements that could be made to the testing procedures which could possible give greater difference in impedance between the two tissues 


\section{ACKNOWLEDGEMENTS}

I would like to thank the Cal Poly Biomedical Engineering Department for their help, support and the knowledge that they have provided me through their classes and office hours. I would also like to thank all my classmates (especially Chris, Lewis, Rolan and Steven) (and friends from Cal Poly, Davis and beyond) who have inspired great conversations about my thesis and for pushing me to think outside the box.

I would like to specially thank Dr. Lily Laiho for her support, patience, understanding and advice throughout my project. I would like to also thank her for providing me with the project and pushing me to go outside my comfort zone. I would also like to thank Dr. Richard Savage for his help in explaining different microfabrication techniques and helping me out even on Saturdays in a clean and having the patience to walk me through things step by step. Dr. Robert Szlavik has been a big help in the thesis, for making all the electrical aspects of my thesis and for making all his classes fun, and always having great discussions about the different facets of electrical engineering.

I would like to also thank Dr. Yarrow Nelson, and Dr. Chris Kitts for helping me understand a lot about microbiology and biochemical engineering. I would especially like to thank Dr. Nirupam Pal for his "punctual" and "professional" behavior which helped me steer myself to Biomedical Engineering.

Lastly I would like to thank my parents and my sister for their love and support and never letting me give up on anything. I would not have been able to complete my thesis if it weren't for their continued support and their continuous encouragement. Thank you to the rest of my family for always being there for me. DD171 


\section{Contents}

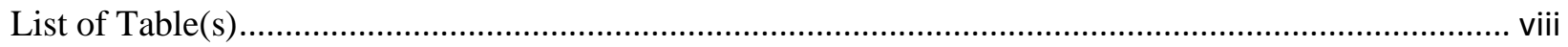

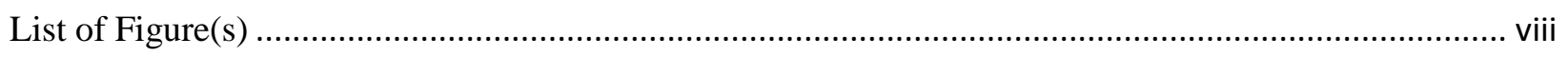

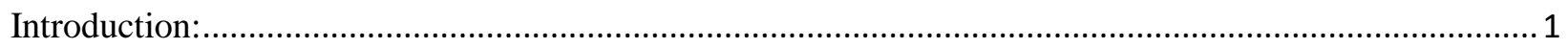

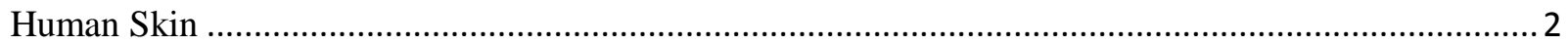

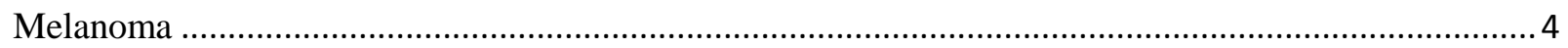

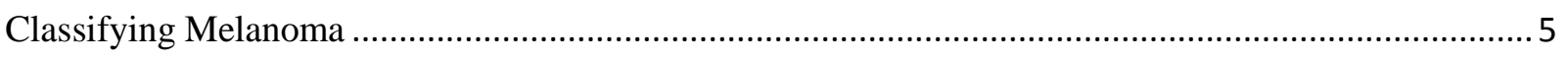

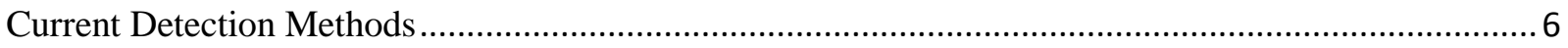

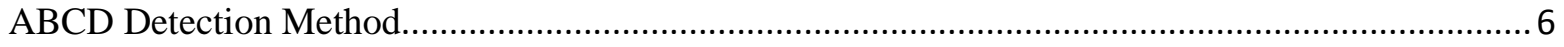

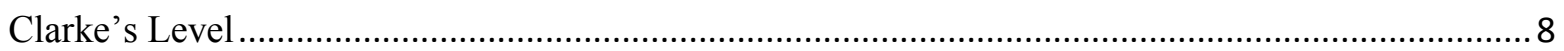

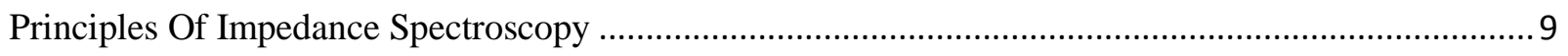

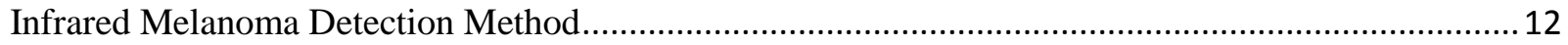

Application Of Impedance Spectroscopy To Melanoma Tissue.......................................................13

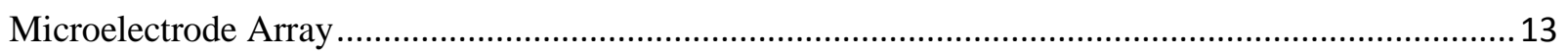

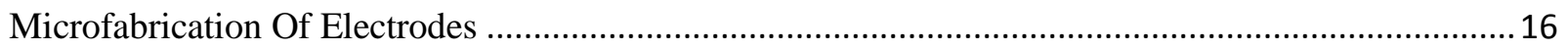

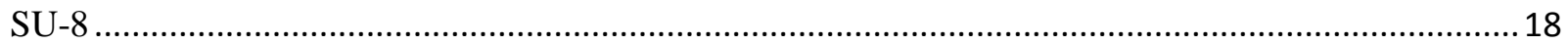

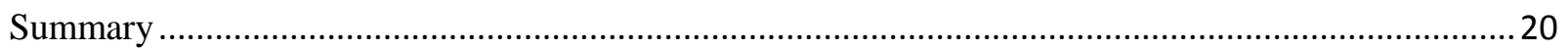

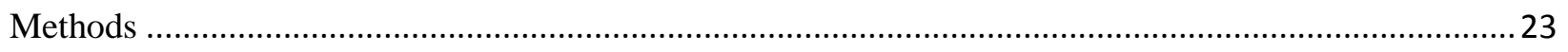

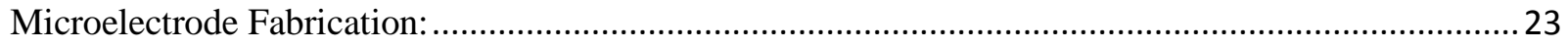

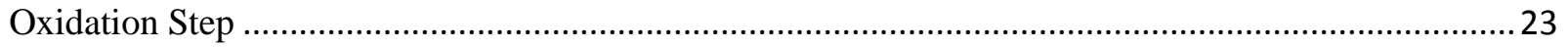

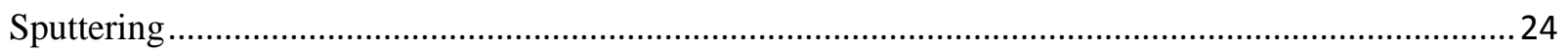

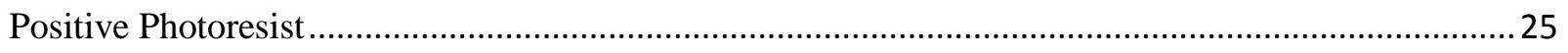

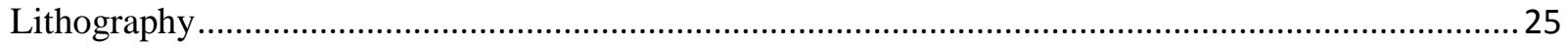

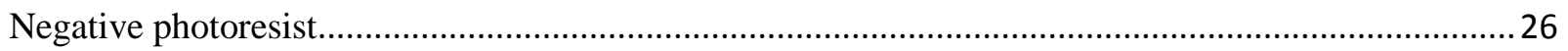

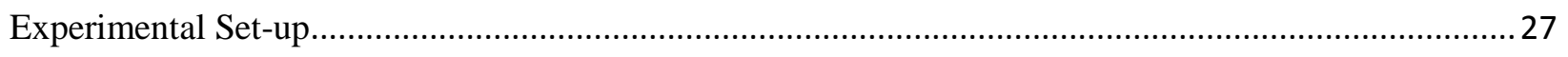

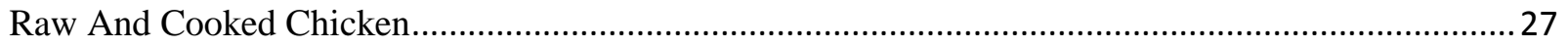

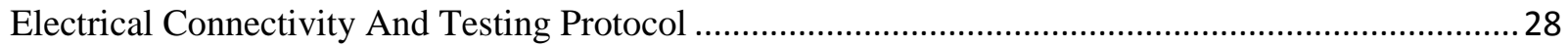

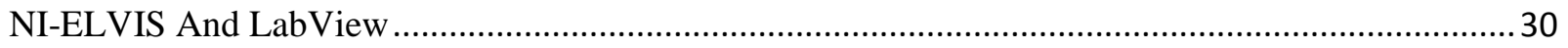




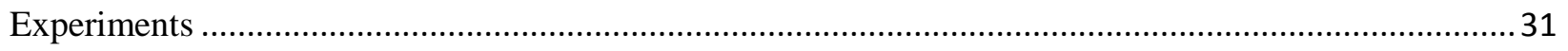

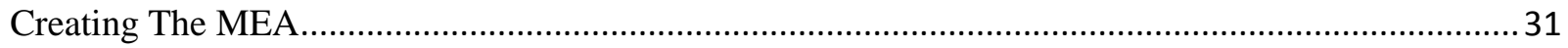

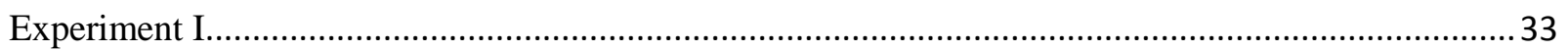

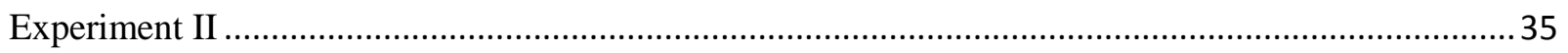

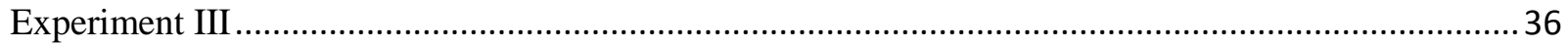

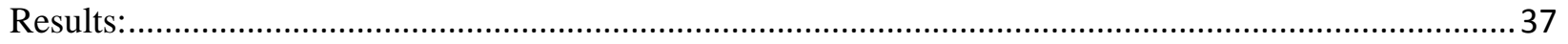

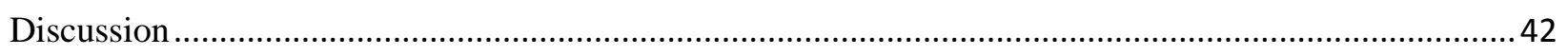

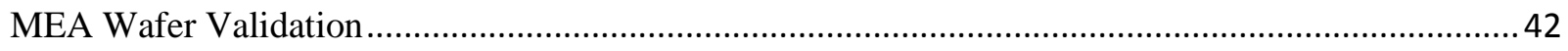

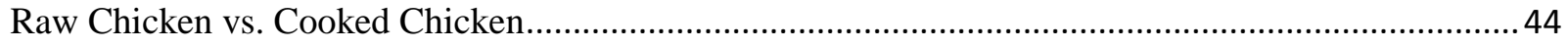

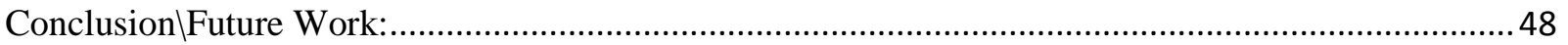

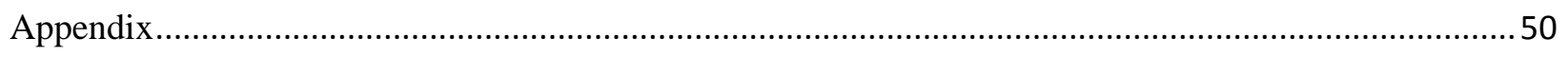

References 


\section{List of Table(s)}

Table 1. Resistance between electrodes.

\section{List of Figure(s)}

Figure 1. Different layers of skin from a histology [4].

Figure 2. Picture showing the different layers of skin with different types of cells present in the epidermis layers. [5]

Figure 3. ABCD criteria for melanoma detection [8] ................................................................ 7

Figure 4. Superficial spreading melanoma with unorganized melanocytes present as single cells and

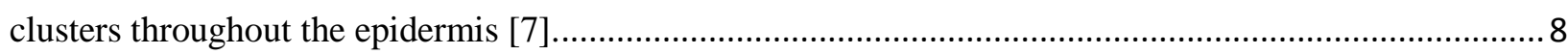

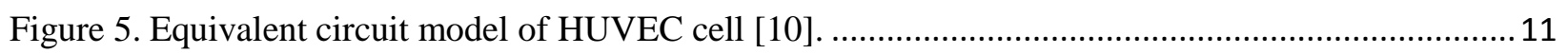

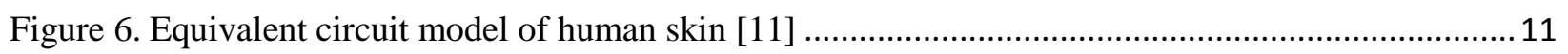

Figure 7. MEA designed for neural recordings [17] ................................................................... 15

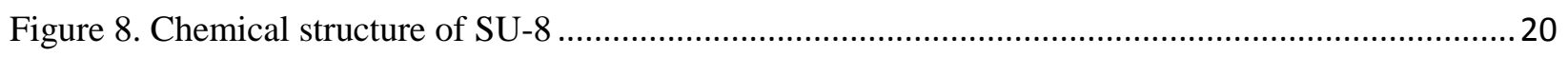

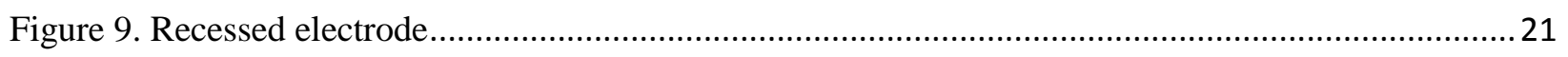

Figure 10. Positive photoresist mask used for MEA with electrodes used in experiments ....................... 31

Figure 11. Finished MEA after fabrication process.......................................................................... 32

Figure 12. Chicken on microelectrode with weight on the chicken while being microprobed...................33

Figure 13. Experimental set-up with microprobe, NI-ELVIS, probe station and chicken for measuring

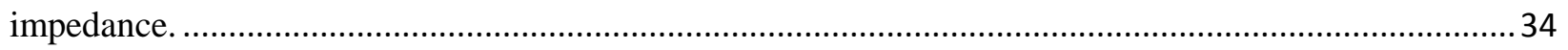

Figure 14. Three electrode set-up used in Experiment II by using three probers and the impedance

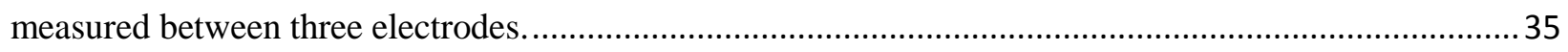

Figure 15. Impedance measurement taken of the raw and cooked chicken (with and without weights) data over 2500, 5000, 7500, 10000Hz. Electrodes E1 and E2 used (as shown in Figure 10)....................... 37

Figure 16. General Linear Model: Impedance versus Frequency, Cook, Weight................................... 38

Figure 17. Impedance measurement raw vs. cooked chicken with 3 electrodes but only 2 electrodes were

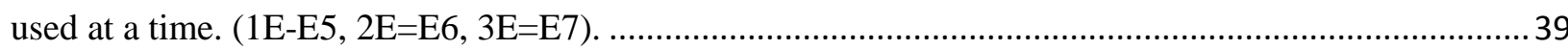

Figure 18. R-Output summary of a Tukey HSD test that looks at statistical differences in frequencies applied between various electrodes............................................................................................ 40

Figure 19. R-Output summary of a Tukey HSD test that shows the stastical differences in raw vs. cooked chicken ( $\mathrm{R}$ is raw and $\mathrm{C}$ is cooked), the variable is represented by temperature, and its impedance

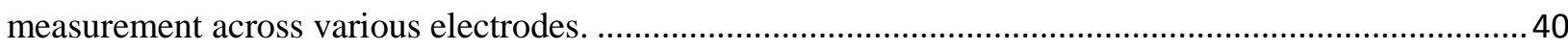

Figure 20. Impedance measurement of raw vs. cooked chicken with an inter-electrode distance of $4 \mathrm{~mm}$

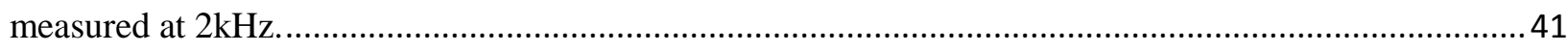

Figure 21. Minitab output of the t-test result for raw chicken (R) vs. cooked chicken impedance (C), at a

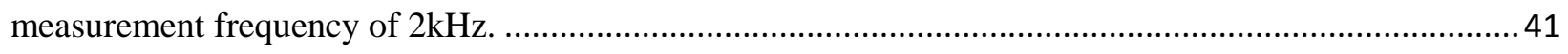

Figure 22. General Linear Model: Impedance versus Frequency, Cook, Weight...................................51

Figure 23. LabView code for Experiment III.............................................................................. 51 


\section{Introduction:}

Melanoma is the fastest growing form of cancer in the United States [1]. In 200559,000 cases of invasive melanoma were reported and about 46,000 new cases of melanoma were diagnosed in situ in the United States [1]. It is the most common type of cancer in women between the ages of 25-29 and the risk of developing melanoma has been steadily increasing [2]. Melanoma incidence rates have gone up from 1 in 1500 for someone in the early 1900s, to 1 in 100 for people born in 1990s and for people born in 2005 it has shot up to 1 in 65 [1]. Melanoma is characterized by uncontrolled growth of skin cells that invade and damage healthy tissue. It is the most serious form of cancer and begins in melanocytes which are responsible for producing the melanin pigment (melanin is responsible for skin color). The cells can spread to the lymph nodes and move to other parts of the body causing damage to vital tissue. Doctors identify melanoma by looking for a lesion which is defined as any abnormality in the tissue (in this case the tissue being the skin). The early detection of melanoma is extremely important to prevent cancer from metastasizing. Currently methods used to diagnose melanoma are based on 'ABCD method' as well as biopsy measurements, which are dependent on the experience of the doctor or lab technician because the detection method requires the lesion to have "irregular border" and the degree to which it can be considered irregular is open to interpretation. Biopsy samples used for histology samples can be a painful experience for the patient due to the fact that a large gauge biopsy needle is inserted into the suspect lesion and a small amount of tissue is removed. A noninvasive method needs to be developed in order to minimize pain for the patient but has to be quick and sensitive enough to pick up the smallest cancerous lesion on skin. In order to fully understand how melanoma works a better understanding of the human skin is needed. 


\section{Human Skin}

The main function of skin is to provide a protective and semi-permeable barrier in order to sustain terrestrial life and avoid desiccation [1]. The prevents water from escaping from our body and helps to keep foreign

$\mathrm{n}$ agents away from internal organs present in the body [3]. It is a semi-permeable membrane allowing certain types of agents to seep through the body to reach the organs such as water, ethanol, or other chemical compounds but blocking other things such as bacteria [2].

The skin is divided into five layers of epidermis and a layer of dermis. The epidermis consists of Stratum corneum, Stratum lucidium, Stratum granulosum, Stratum spinosum, Stratum germinativum (Stratum basale), and the dermis layer [1]. Stratum basale is made up of cells that have a cube like or column like appearance and are closest to the dermis layer [1]. Stratum spinosum layer consists of a high concentration of desmosomes (cell structure responsible for cell to cell adhesion) and keratin filaments (fibrous protein used for structural purposes) resulting in its spiny appearance [1]. The next layer is the stratum granulosum which have keratohyalin granules, protein found in granules, bound to keratin filaments which result in the formation of very compact and dense structures in the cytoplasm causing a granular appearance of the layer [1]. Stratum corneum is the outermost layer of the skin and is the point where keratinocytes (the predominant cell type present in the epidermis) have lost all their nuclei and organelle and have become elongated and flattened [1]. These cells form an array of cells called "corneocytes" which are held together by the remaining desmosomes (desmosomes are cell structures specialized for cell to cell adhesion) and intracellular excretion from Odland bodies, organelles that contain cementing like compounds[1,2]. 


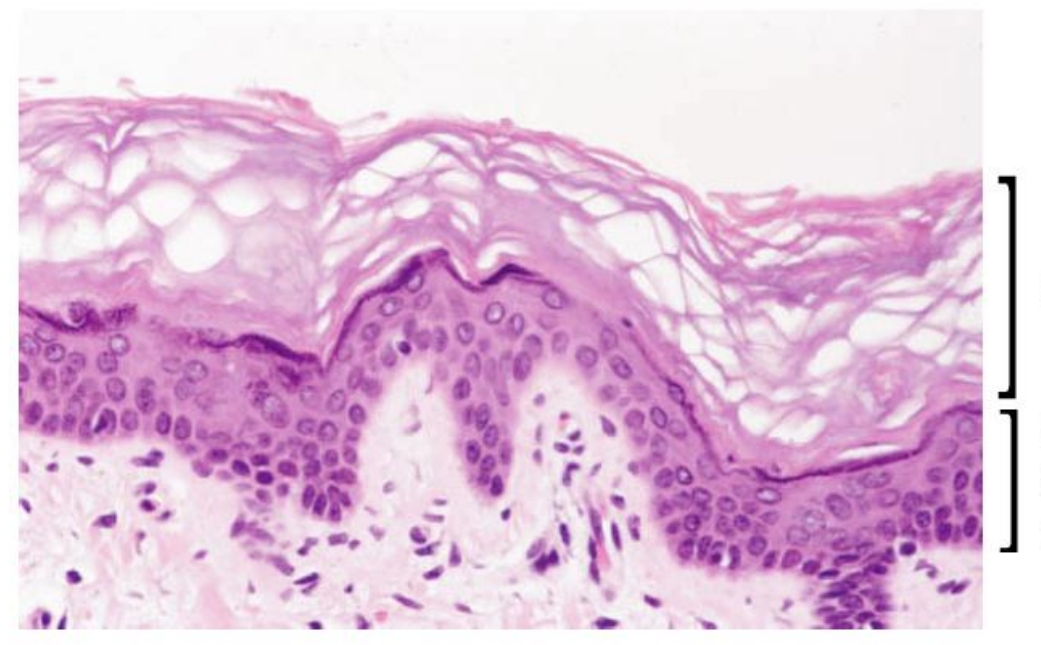

Stratum corneum

Stratum granulosum

Stratum spinosum

Stratum basale

Figure 1. Different layers of skin from a histology [4].

Keratinocytes are the most abundant type of cells present in the epidermis [2]. As keratinocytes travel upwards through the epidermis they begin to produce keratin filaments, a major contributor to skin toughness, and simultaneously begin to die and lose their organelles in a process known as keratinization [1]. The second most abundant type of cell is a melanocyte which is a type of dendritic cell (cell that is part of the immune system) found in the stratum basale [1]. Melanocytes are responsible for synthesizing and secreting organelles called melanosomes which contain melanin [1]. Melanin is a pigment that is found everywhere in nature and the type of melanin produced and their distributions in the surrounding keratinocytes are responsible for the various skin colors [1]. Melanocytes are also responsible for darkening of the skin due to UV radiation from the sun and are involved in a process called apocopation in which melanocyte cells transforms to keratinocytes $[1,2]$. The next most common cells are the Langerhans cells which are an antigen presenting cell derived from the bone marrow and perform the function of surveillance for the immune system [1]. 


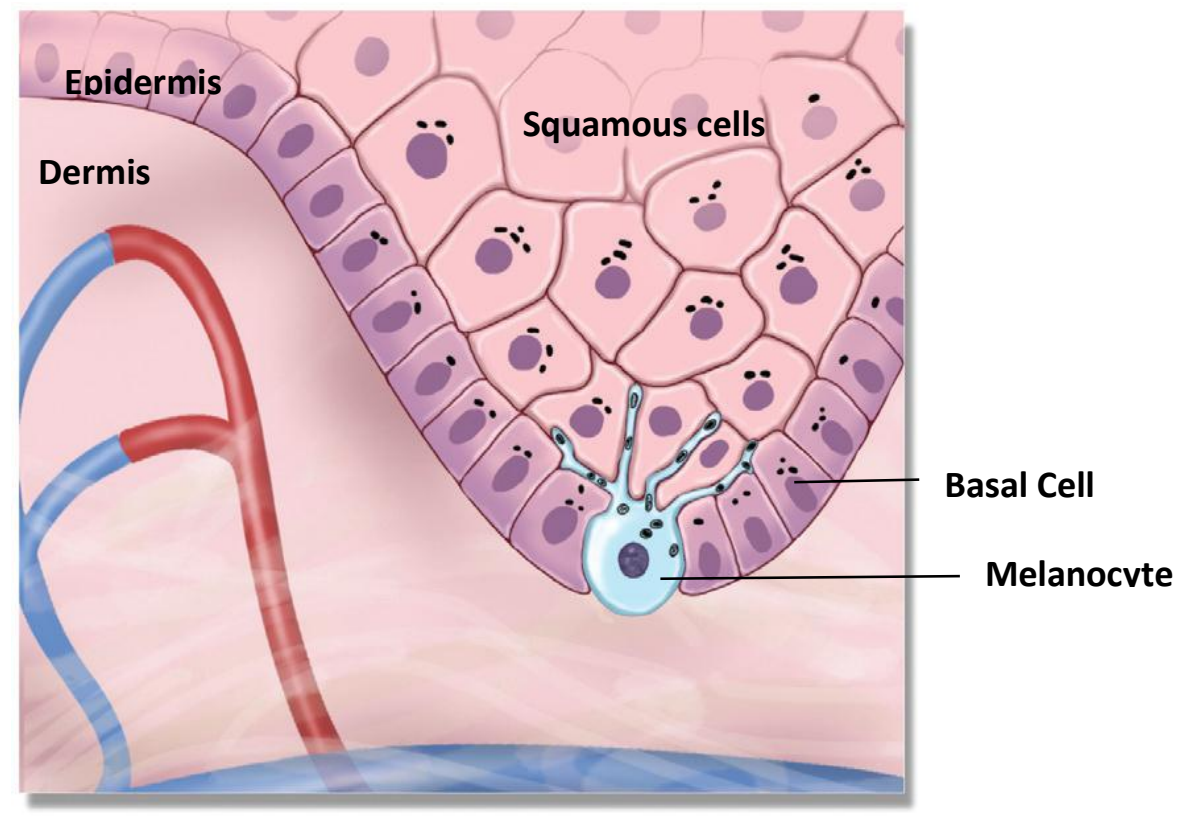

Figure 2. Picture showing the different layers of skin with different types of cells present in the epidermis layers. [5]

\section{Melanoma}

Skin cancer can be placed into four categories, which are malignant melanoma, basal cell carcinoma, squamous cell carcinoma and actinic keratosis [2]. Of these cancers basal cell carcinoma and squamous carcinoma are considered non-melanoma skin cancer. Melanoma is caused by the malignant melanocytes and nevus cells [1]. Nevus cells are the cells present in a localized malformation called cutaneous hamartoma (harmless proliferation of cells) [1]. Nevus cells can become malignant and in the case of malignancy these cells are just referred to as Melanocytic nevus cells. It has been suggested that brief intense exposure to long wave UV radiation or the loss/mutation of tumor suppressing genes can cause melanoma[1]. The groups at greatest risk of developing melanoma are people with fair skin that are exposed to sunlight in short intense durations of time, immediate family member diagnosed with melanoma, and have many uncharacteristic appearing nevi [1]. There is a harmless version of these lesions called the 
Benign nevus that can sometimes be mistaken for melanoma [6]. Melanoma identification can be difficult, so some sort of classification method(s) are needed to verify the tumor.

\section{Classifying Melanoma}

Melanoma is subdivided into many groups which include superficial spreading melanoma, Nodular melanoma, Lentigomaligna melanoma, Acrallentiginous melanoma and Amelanotic melanoma [1]. Superficial spreading melanoma is the most common type of melanoma and represents $70 \%$ of the melanoma cases [2]. Specifically, superficial spreading melanoma spreads along the epidermis and will enter the dermis after several years [2]. Nodular melanoma represents $15 \%-30 \%$ of all melanoma cases [2]. This type of melanoma goes through the epidermis and dermis layer faster as compared to other melanomas [2]. Superficial spreading melanoma can be detected by darkening of pre-existing moles [2]. Nodular melanoma is usually a pigmented papule that grows and can cause constant degeneration of the tissue at the site of melanoma [7]. Lentigomaligna melanoma usually arises from a pre-existing skin lesion that has been photo damaged (also known as a lentigo) and is responsible for 5\% of melanoma cases [3]. Lentigomaligna usually appears large, flat, and contain tan colored lesions whereas other melanomas can have different colors such as pink [7]. It is another subtype of melanoma and is found in elderly people that have very sun damaged skin [7]. It does not have the characteristic uneven spreading of melanoma cells as is present in superficial melanoma. The epidermis around Lentigomalgina is seen to be in a state of decay. The lentigomaligna melanoma cells come together into one cluster usually along the dermal and epidermal junction. These cells tend to be very densely packed and small[7]. Acrallentiginuous melanoma is the most common form of melanoma for African Americans and Asians [2]. It is usually found on the palms of the hand, soles of the feet, and can also be found under the nails and appear as brown or black changes in 
the skin color that are neither raised nor depressed (also known as macules) [2]. It can be sometimes be mistaken for a bruise which can result in this type of melanoma being diagnosed much later[3]. Amelanotic melanoma is a non-pigment producing form of nodular melanoma and can sometimes be confused with benign skin lesions [1]. The categories discussed above allow for melanoma classification by the doctor and histology but a truly quantitative method is needed. Hence, doctors have discovered ways to quantify melanoma and its extent of spreading.

\section{Current Detection Methods}

\section{ABCD Detection Method}

The current detection method that is used is called the ABCD method [2]. In this method a malignant melanoma is considered if it has : $\mathrm{A}=$ Asymmetry, $\mathrm{B}=$ Border irregularity, $\mathrm{C}=$ Color variation, $\mathrm{D}=$ Diameter greater than $6 \mathrm{~mm}[2]$. The suspected melanoma is then excised and viewed under a microscope via histological slides to more accurately determine if the excised tissue is truly contains cancerous cells [2]. The ABCD detection method is subjective and varies from doctor to doctor. 


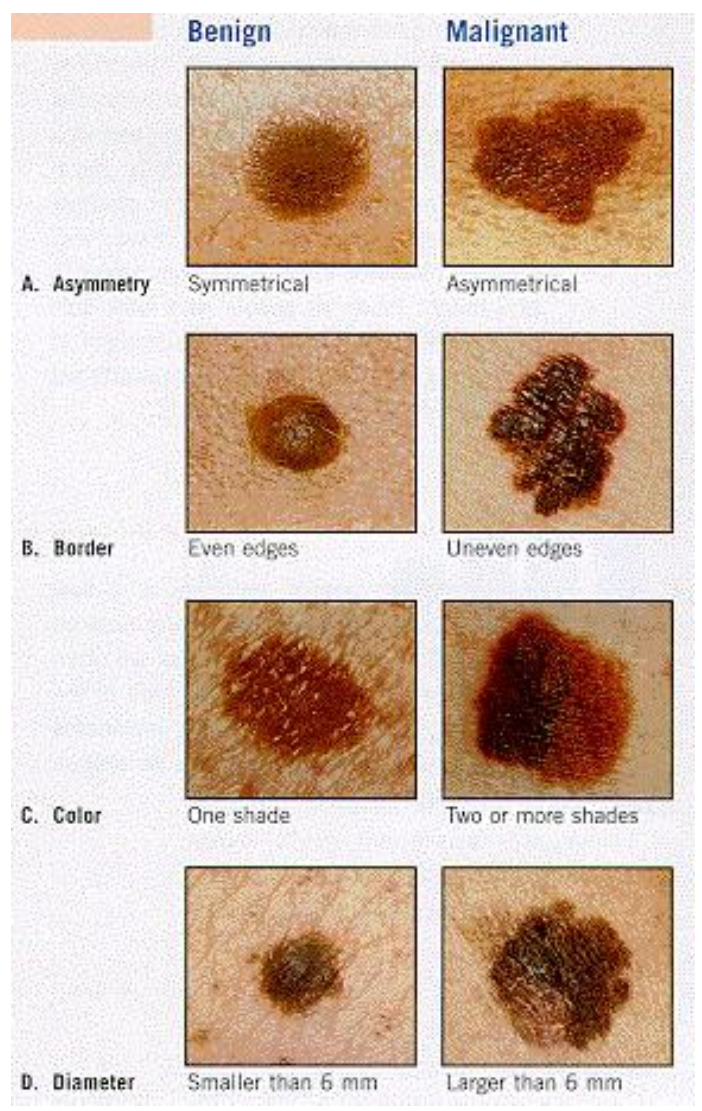

Figure 3. ABCD criteria for melanoma detection [8]

Figure 3 shows the differences between what is considered a malignant or benign, the extent of malignancy as shown above can be subjective from doctor to doctor. Once the dermatologist has excised the tissue via a skin biopsy, the tissue sample is stained at a histology lab [6]. Staining the cells allows the histologist to look for signs of malignant melanoma as shown in Figure 4 [6]. In the case of superficial spreading melanoma which is usually the initial indicator of malignant melanoma is identified by lateral spreading of malignant melanocytes through the epidermis and by their unorganized distribution through the epidermis [6]. Malignant melanocytes also tend to have clusters of cells that seem to be "falling apart". Once the malignant cells move to the dermal part of the skin the malignant cells can be identified by their mitotic activity, immature cells, inflammatory response, and neovascularization (formation of micro vascular networks). 


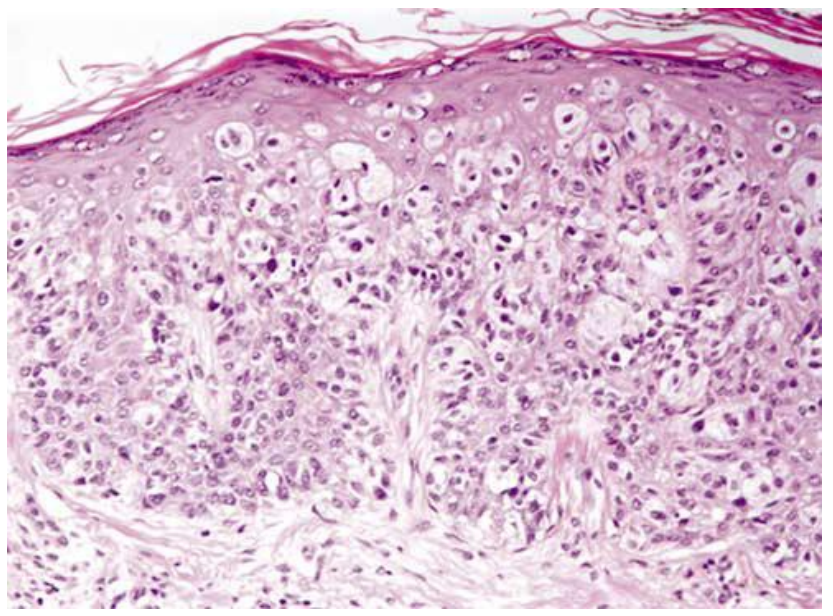

Figure 4. Superficial spreading melanoma with unorganized melanocytes present as single cells and clusters throughout the epidermis [7].

\section{Clarke's Level}

Clarke's levels as described by Dr. Wallace H. Clark, Jr. is a measurement system used to describe the invasion depth of melanoma which can be used as a way to understand the development of the disease and also can correlate with mortality rates of the patient [1]. This method can be used in conjuction with the ABCD method in order get a better understanding of extent of the melanoma. Level I is defined as the tumor cells are only present in the epidermis [1]. Level II is extension of tumor cells from the epidermis into the papillary dermis (uppermost layer of the dermis) but has not yet filled the papillary layer [1]. Level III is when the tumor cells instead of just reaching the papillary layer in Level II, the cells have actually filled up the papillary layer [1]. Level IV is when the cells have reached into the layer below the papillary layer, also known as the reticular dermis [1]. The last level is Level V where the tumor has gone through the epidermis and dermis layer and has reached subcutaneous fat [1]. The other measurement technique is known as Breslow's depth which uses an ocular micrometer to measure the invasion depth from the top of the granular layer to the farthest invasion depth of the 
tumor and it usually done using a microscope[1]. The skin sample is usually acquired through a biopsy of the tissue in question[1].

The current diagnostic techniques require histologic staining of the tissue which increases the time in getting the results back and also increases the cost of testing. A diagnostic method is required that can detect melanoma quickly and accurately without the necessity of histologic staining and decrease detection time. An earlier detection time also has its benefits, it is estimated that if melanoma is caught during Stage I of the disease then there is a 90-95\% survival rate but at later stages the five year survival rates drop to 50\%[5]. Detection using impedance spectroscopy could provide a means of accurate and early detection on lesions that would be too small to biopsy and it might be able to have more sensitivity than the current detection methods employed.

\section{Principles Of Impedance Spectroscopy}

The current melanoma detection method can be improved to provide point of care testing for small suspect lesions if impedance spectroscopy can clearly distinguish malignant tumors. This detection could potentially be done by exploiting the differing electrical properties of skin and melanoma. Thus it becomes important to understand the electrical properties of melanoma because they could be used as a method for diagnosing various lesions present on the human. A better understanding of the electrical properties of melanoma and skin could also provide an objective method of melanoma detection instead of the current ABCD method employed by doctors and can be tested on smaller lesions providing earlier diagnosis. It could remove the cost associated with histology staining of the biopsy sample and subsequent analysis done by the histologist to measure the invasion depth of the melanoma cell, only if the impedance differences can be exploited. This detection methodology lends itself to the concept of Impedance or 
Dielectric Spectroscopy. Impedance spectroscopy, more specifically Electrochemical Impedance spectroscopy (EIS) is a tool that can give information about the ionic content of various solutions[9]. Ionic content give information about the amount of ions present in and outside a cell which are extremely important for the survival of the cell and are present in various concentration in the intracellular fluid and the extracellular fluid [9]. These measurements can be an important tool for distinguishing different types of cells, such as nerve cells from epidermal cells or differentiating various tissues [9]. It can also be used to determine if a cell is under stress [9]. The cells are measured under a range of frequencies to differentiate different types of cells and to provide a health status of the cell [9]. This is due to the fact that different types cells have different "equivalent electronic circuitry" which results in different impedance characteristics for cells [10]. The circuitry representative of human skin will not be representative of a HUVEC (Human Umbilical Vein Endothelial Cell) cell[10, 11]. EIS can used to measure the ionic content of intracellular fluid which can also be an important indicator of tissue health [9]. The varying ionic concentration and its effects on $\mathrm{pH}$ could affect the electrical characteristics of the tissue and be a determining factor for tissue health [9]. The higher the ionic concentration the higher the conductance through the cell or tissue as the ions provides a current propagation path [9].

As seen from the Figures 5 and 6 it can be seen that different types of cells will have different equivalent circuits associated with them. This identity could be exploited to distinguish different types of cells and can also be used to distinguish healthy skin cells from malignant melanoma cells. It was shown by Stante (2009) et al. that it is possible to differentiate between healthy skin and melanoma tissue using EIS between two electrodes. The success of Stante's work led to the next step which was the development of an array of electrodes to see if it could 
be used as a technique to create an electrical image of the tissue[12,13]. Impedance spectroscopy is an important tool, but there needs to be an understanding of other detection methods currently being researched and juxtaposing it with impedance spectroscopy.

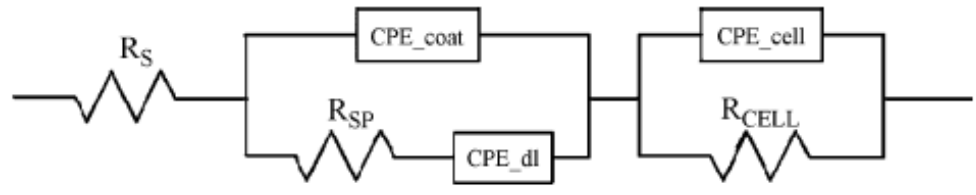

Figure 5. Equivalent circuit model of HUVEC cell [10].

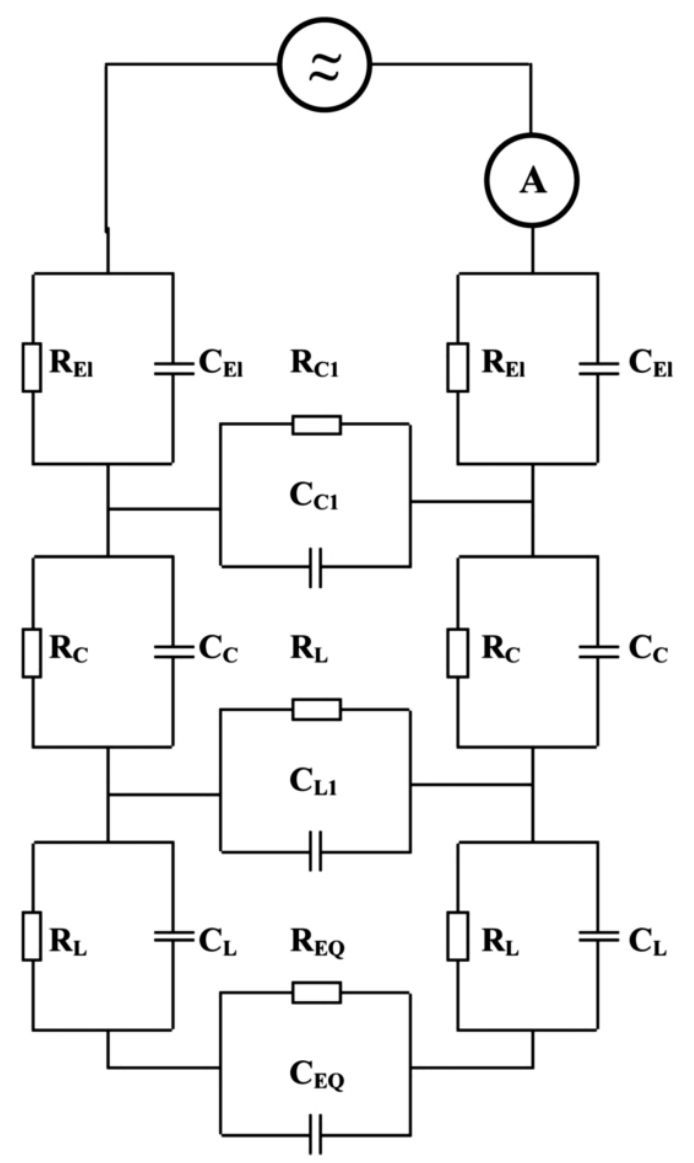

Figure 6. Equivalent circuit model of human skin [11] 


\section{Infrared Melanoma Detection Method}

Separate from impedance sensing there are several technologies being employed for the detection of melanoma. There are several non-invasive methodologies being used to detect melanoma non-invasively. One such approach uses Near Infrared Light (NIR) to determine if the lesion in question is cancerous or benign [14]. NIR is light waves that are between 700$2500 \mathrm{~nm}$ and in these applications the measurement is done across many frequencies [14]. As NIR light is shone on the tissue, it causes the some of the light to while some of it is absorbed [14]. The correlation between scattered vs. absorbed light for tissues can be used as a tool to determine if the skin tissue is cancerous or benign [14]. This could prove to be a useful noninvasive method if it is employed as it uses the absorbance of the NIR light to determine the different types of tissues [14]. There are however drawbacks with this technique. Since this is an optical imaging technique it requires the patient to be extremely still which can be quite difficult for some patients especially the elderly ones [14]. A lack of understanding in the NIR spectra of tissues could cause problems to the person diagnosing because it could be that certain biochemical reactions could have similar NIR spectra [14]. Thus a greater understanding of NIR tissues interaction is needed[14]. Many of the optical techniques require an understanding of the interaction of tissue with optics and can require extensive image processing ability as well as a finely tuned optical system [14]. It can also be difficult because movement of the person can cause distortion of the images which can lead to erroneous analysis and the difficulty for patient to remain still during image acquisition [14]. Impedance spectroscopy doesn't use light as its medium of detection but uses current instead to make measurements. It avoids using optics but instead requires more signal processing. Thus, the next step would be to apply impedance spectroscopy for melanoma detection. 


\section{Application Of Impedance Spectroscopy To Melanoma Tissue}

Impedance spectroscopy is a non-invasive method of measuring melanoma and if proven could be easily implemented due to its ease of use and reduced cost. There has been research into impedance spectroscopy of skin cells to detect melanoma cells. Previous research such as Glickman (2003) et al. showed that impedance spectroscopy was able to classify human melanoma lesions with $92 \%$ sensitivity and 67\% specificity[15]. Stante (2009) et al. also successfully reiterated the concept of being able to detect melanoma using impedance spectroscopy to distinguish between melanoma and healthy skin tissue[13]. Aberg (2004) et al. showed that a multi-frequency approach to detecting melanoma could differentiate malignant melanoma with $89 \%$ accuracy[6]. This implicated the ability to use multiple frequencies ranges to more accurately detect melanoma while as in Glickman (2004) et al. only one or two frequencies were used, such as $2 \mathrm{kHz}$ and Fleshman (2011) et al. used 2kHz and 10kHz. Stante (2009) et al. and Fleshman (2011) et al. used large Ag/AgCl wires which showed the ability to measure the impedance differences between electrodes for melanoma vs. healthy skin tissue[12, 13]. Using wires can distort the image because the impedance gets integrated over the size of the sensor resulting in an averaged impedance value in that area. If a $1 \mathrm{~mm}$ diameter wire is being used, the resulting output will be the average impedance over the area of the wire. This could lead to erroneous information when considering that certain lesions can be as small as $6 \mathrm{~mm}$. Small electrodes are needed in order to reduce the area the tissue is averaged over and such small areas are only possible with the use of semiconductor techniques by developing a microelectrode array.

\section{Microelectrode Array}


Microelectrode arrays (MEAs) have been used quite widely for neural recordings in literatures. Microelectrode arrays are an array of electrodes that are placed very close together to provide detection of signals from very small points [16]. The electrodes detect event that can be electrically detected such as currents caused by ions. MEA also gives measurements from a finer point since the signal is no longer being integrated over a large area [16]. It can be used in electrically detecting objects as small as cells such as neural cells, a sample MEA is shown in Figure 7 [17]. The impedance characteristics of the neural cell are recorded under various cellular conditions to see how signal transduction changes through the neural pathway and can also be used to measure action potentials in neural cells or muscle cells[17]. MEAs are starting to find uses in various areas throughout literature and novel methods are being developed for measuring impedance characteristics of certain types of cells or tissues. Recently, work was done on flexible MEAs because they allowed the electrodes to bend and deform to the shape of the tissue [18]. This can be extremely useful for measuring tissue that has amorphous shapes or tissues that can be damaged easily due to stiffness of the electrode (which is usually found on $\mathrm{SiO}_{2}$ substrate). 


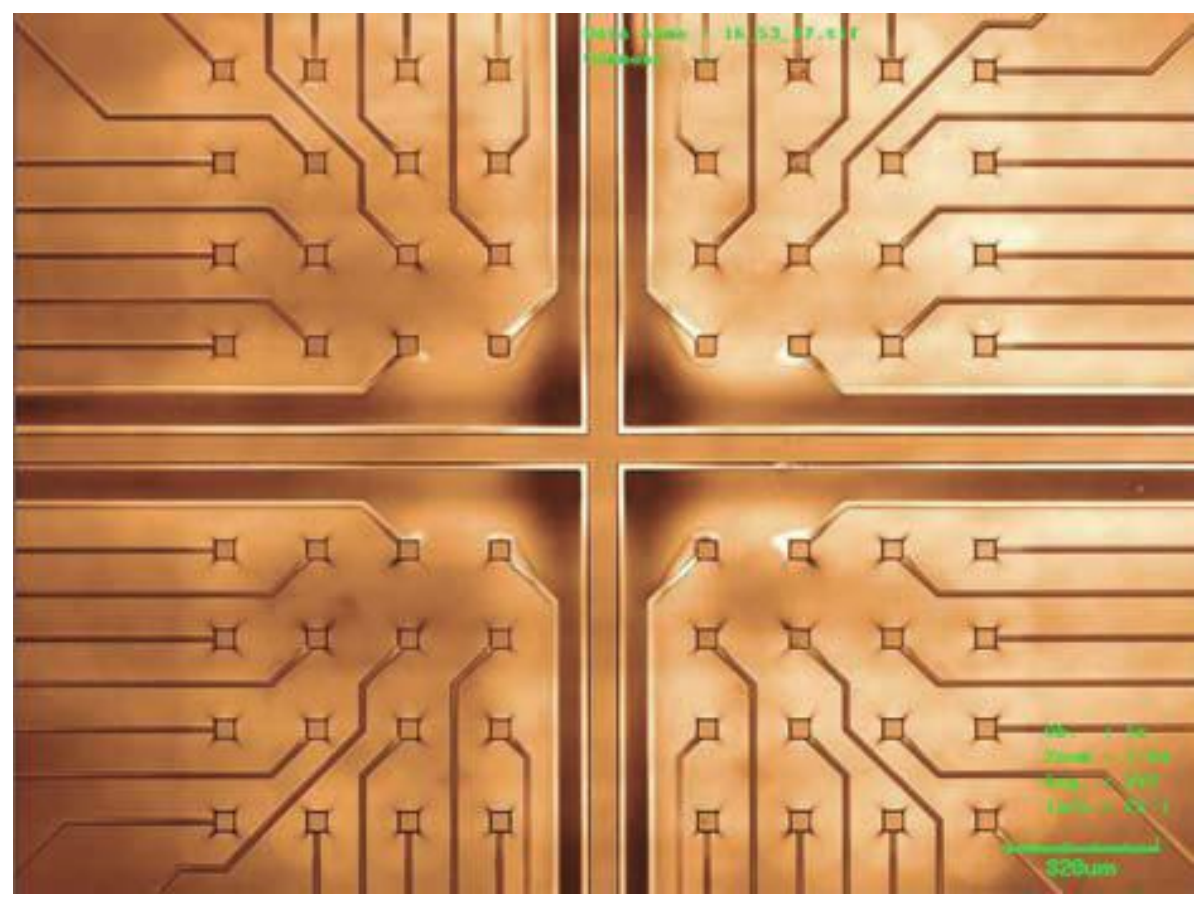

Figure 7. MEA designed for neural recordings [17].

The reduction of size is important for finer measurements of the tumor. These could in the future be laid out in a pattern in order to produce a clinical image to be used by the doctor or to provide spatially information. A discrete sensor " $\mathrm{i}$ " will have a discrete output specific to that sensor which can be developed into a matrix of sensors resulting in impedance info from several directions[16]. These sensors will have discrete measurements and should be able to more accurately quantify the boundary of the tumor which can reduce the amount of tissue excision required upon tumor removal.

Increasing the number of electrodes per unit area allows for a greater sampling area and a much higher resolution. Having electrodes with such a fine pitch (interelectrode distance) is only possible using semiconductor fabrication methodology where photolithography can bring the size of electrodes down from $1 \mathrm{~mm}$ to $0.25 \mathrm{~mm}$ or smaller. This is beneficial because it allows for smaller electrode area and allows detection of extremely small lesions (on the order of microns). 


\section{Microfabrication Of Electrodes}

Microelectronic is usually reserved for technologies related to the semiconductor process and are usually done on the micron scale [16]. The benefit of using this process is to have very small sample reactions to reduce the amount of analyte that is needed to get a diagnosis or measurement or to take readings from extremely small sample area [16]. The only widely available process that can be used to make MEAs (microelectrode array) is available in semiconductor device fabrication since the production of transistors and solar cells is done on the order of microns [16]. The semiconductor fabrication process is well understood for the micron scale and can be applied to the biological world with minimal processing modifications. Microelectronic devices made in industry require a clean room which can be expensive in terms of capital but are able to produce millions of devices per year with very small feature size and very high reproducibility and reliability [16]. The repeatability and large scale production of features that are on the order of microns make this process a smart choice for creating a microelectrode array and aid in driving down the cost of the electrodes. Conventional photolithography techniques can produce smaller electrodes thus allowing for greater resolution and new technologies are constantly developing to allow for features sizes down to nanometers.

The basic processes used in semiconductor fabrication are oxidation, sputtering, wet etching and photolithography. Oxidation involves the growth of an oxide layer on top of a Silicon substrate usually done at high temperatures the use of steam to split the hydrogen and oxygen molecules present in water. The oxygen molecule then bonds to the Silicon to create Silicon dioxide. Sputtering is used for placing a couple nanometers thick layer of metal on top of some substrate such as silicon or silicon dioxide. This can be done using physical vapor deposition where ionized gas such as argon hits a gold target causing the gold molecule to ionize 
which then releases a gold atom. The ionized gold atom travels to the Silicon wafer due to a electrical potential created inside the sputtering machine. Wet etching is used to create traces and to remove any unwanted metal layers in order to create electrodes and wire traces. This process usually involves a photolithography step in which a polymer called photoresist is placed on silicon wafer. The photoresist is polymerized along certain areas by UV-light with the usage of a mask which defines the polymerized patterns. The positive photoresist solubilizes in a photoresist developer when exposed to UV light where as a negative photoresist polymerizes when exposed to UV light [17]. After exposure, the wafer is placed in a developer solution and the region that can be solubilized easily dissolves in the developer solution only leaving behind the polymerized photoresist [17]. The region which has no photoresist is now open for any chemical wet etching step since the metal is exposed [17]. The wafer is then placed in an etching solution where all the regions not containing the photoresist are attacked and that metal is etched or eaten away [17]. Once the etching process is done the only remaining metal is the metal that was under the polymerized photoresist [17].

The process of photolithography, oxidation, and etching is very well understand and used quite frequently in semiconductor industry, if diagnostic devices could be built on this paradigm it could provide instruments very cheaply. Lower cost would mean access to more hospitals and doctors to reduce the incidence of misdiagnosing the lesions. The electrode being designed in this report will be akin to conductometric biosensors which are simply the measurement of impedance between two electrodes [16]. The electrodes measure the change in conductivity through the solution (or tissue) by applying various frequencies and seeing the change in conduction. In the case of conductometric biosensors the impedance is measured and a reaction occurs between two electrodes which causes a change in conductance which is then picked up by 
the electrodes [16]. These sensors can be made to very sensitive and are used in sensing urea, Lasparagine and creatinine [16].

\section{SU-8}

Since the electrodes are on semiconductor wafers, an electrical insulator is required to isolate the electrical traces on the wafer from the skin so as to not effectively increase the electrode area. The photoresist needs to be inert to various chemicals especially isopropyl alcohol and ethanol which are frequently used as disinfectants. The insulator needs to be cheap, and readily available and be biologically inert.

SU-8 is an epoxy which is a thermosetting plastic created by a reaction of a resin and a hardener as shown in Figure 8 [19]. The resin part of the molecule bonds to the surface such as Silicon and the hardener is the material that polymerize after exposure to UV light and subsequent baking process [19]. When thermosetting plastics are heated it causes the plastic to irreversibly cure (cross-link) while at the same time the SU-8 bonds to a metal layer underneath the SU-8, such as gold which could be on top of a silicon wafer [19]. SU-8 is a negative photo resist (a material that is sensitive to light, and is frequently used in photolithography in situations such as semiconductor fabrication) which means that the portion of the photo resist that is exposed to UV-light will become insoluble in a solution of the photo resist developer and the region that is not exposed will be dissolved away by the developer [17]. SU-8 was developed by IBM for the microelectronics industry but has since found its place in MEMS and bio-MEMS due to its unique properties and its biocompatibility (Biocompatibility is how well the material interacts with biological materials) [20]. One of the features of SU-8 is its inertness with most chemicals that it interacts with [17]. Along with this is its ability to prevent biofouling (the ease with which cells can bind on to the surface of a material) on its surface and its ability to be 
biocompatible since it does not react with the common radicals found in the human body [20]. The advent of bio-MEMS has allowed SU-8 to be used for culturing cells, as possible medium for bio-MEMS drug delivery systems and but not limited to a force transducer in the soft tissues[18,20]. SU-8 also performs well electrically and can act as an electrical insulator allowing for circuit isolation [17]. This can be attributed to its high dielectric break down voltage, and its ability to insulate even at high frequencies (up to $10 \mathrm{MHz}$ ) which is useful in dielectric spectroscopy[18, 21].

SU-8 is a well characterized, well understood and widely used in industry allows it to be easily implemented in most clean rooms [21]. Curing of SU-8 is a routine semiconductor process which further supports the implementation ease of the MEA. Its background in microelectronics industry has made it a cheap and effective alternative. Ease of implementation in manufacturing and biocompatibility allow it to be easily used in bio-MEMS or MEMS devices [18]. SU8 has been used in literature as a method of electrically insulating cells in an microelectrode array and it was seen that SU-8 had no effect on the neural cells[17]. SU-8 has insulative properties which can be used to electrically isolate different electrodes that doesn't interfere with the biochemical reactions of the body and doesn't deleteriously affect the body. In the design of MEA for impedance testing the SU-8 will be used for providing electrical isolation and chemical inertness with its environment. These uses will be to create an electrical isolation between the traces and the skin and it will be used due to its biocompatibility. 


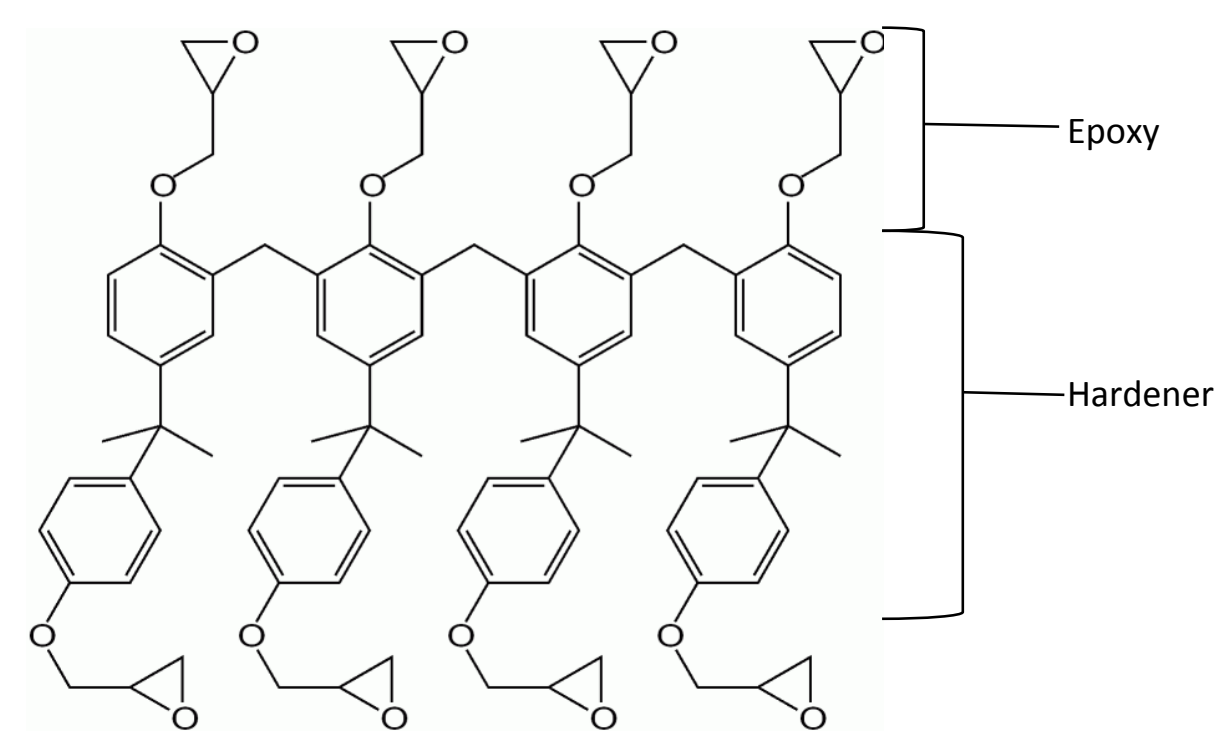

Figure 8. Chemical structure of SU-8

\section{Summary}

These methodologies when implemented into a sensor can be a quick and powerful tool for diagnosing melanoma. If the detection system is accurately able to detect melanoma then its ease of manufacturing due to its cross compatibility with semiconductor fabrication make it a cheap and accurate diagnosing device to give the doctor the most cost effective tool to identify suspect lesion. This technique does not depend on optics and doesn't require heavy image processing algorithm which can be an added cost and time for the doctor implementing such programs. Success of simple diagnostic techniques provides an objective way to measure lesions quickly and effectively without causing pain and discomfort to the patient as is the case of needle biopsies. The thesis will look into detection of two different types of tissues (cooked chicken and raw chicken) using impedance spectroscopy and will try to show that the cells can be differentiated based on their respective impedance. If successful this tool will bring the possibility of using impedance spectroscopy a step closer to implementation in the real world which could be beneficial for fast spreading cancers such as melanoma. The testing will look at the impact of electrode diameter and its ability to differentiate between cooked and raw chicken 
and the effect of electrode-electrode distance on accurate detection. Along with all this, it will be also tested to see if recessed MEA (as shown in Figure 9), that are below the insulative SU-8 layer, can be used for the detection of melanoma cancer cells.

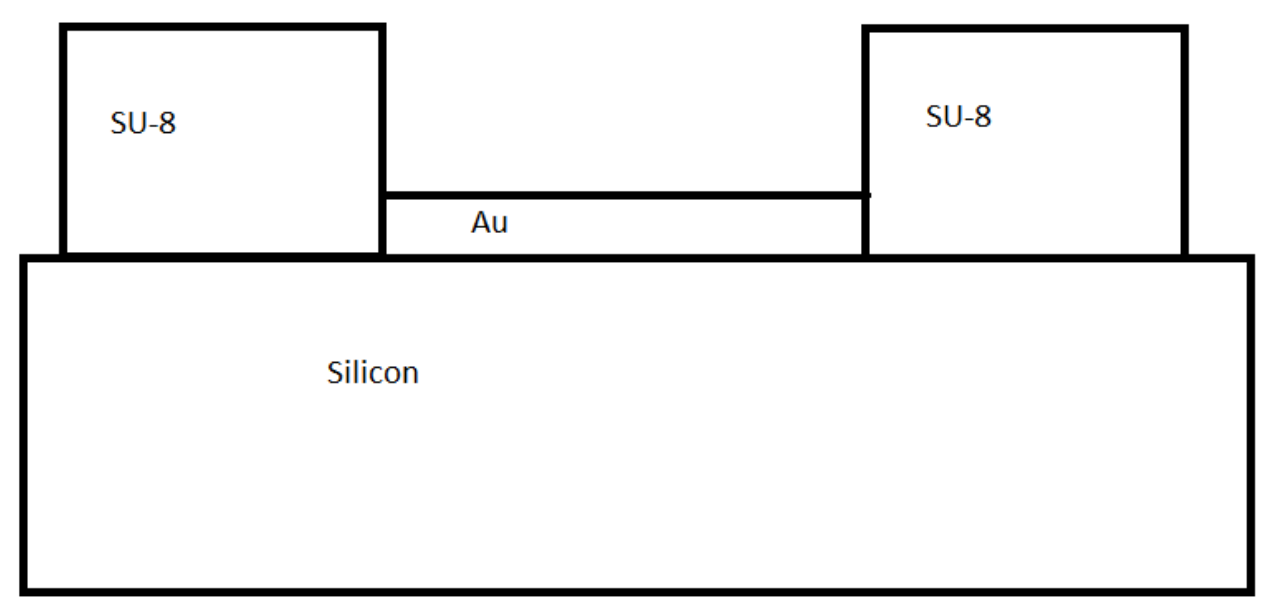

Figure 9. Recessed electrode

These tests will determine if EIS has a potential in determining melanoma cells vs. healthy skin cells. It could also used for determining other types of skin cancer and for determining other cancers. The added benefit of using this technique would be that it would cheaply available due to its simplicity in creating the MEA and the detection method uses current which would reduce cost of the device, and a shorter time to get results and allow for earlier diagnosis for melanoma.

The next step based on the success of this design would be to see if the sensor also works on melanoma vs. healthy skin tissue and after that making the device portable. The visual representation is necessary because it allows for the doctor to view what is going on at a micron level and gives the doctor an image similar to a topographical map. The accuracy of the image would be a function of how close the electrodes are to each other, because the closer the electrodes are the better resolution the "images" will have. It would also be interesting to see the effects of different metals other than gold on measuring impedance across various tissues. Electrodes made of $\mathrm{Ag} / \mathrm{AgCl}$ would also be an interesting next step, due to the fact that $\mathrm{Ag} / \mathrm{AgCl}$ 
electrodes are non-polarizable and can be constantly reused which can be important in terms of cost savings provided the proper sanitization technique is used to clean the electrodes. The goal of this thesis is to show that accurate measurements can be made at very small distances between electrodes and that the electrodes can pick up the variation between tissue of varying ion content, such as melanoma vs. healthy skin tissue. 


\section{Methods}

\section{Microelectrode Fabrication:}

A 6" n-type silicon wafer was used as the first step in the fabrication of the microelectrode. The wafer had several sensors placed on it with electrode diameters of 50, 100 and $250 \mu \mathrm{m}$, and the sensor sizes were $0.5 \mathrm{~cm}^{2}, 1 \mathrm{~cm}^{2}$ and $2 \mathrm{~cm}^{2}$. The mask was designed to fit a total of 7 sensors. There was only one $0.5 \mathrm{~cm}^{2}$ sensor (the distance between the bond pads was $0.5 \mathrm{~cm}^{2}$ ) which had an electrode diameter of $50 \mu \mathrm{m}$ and the distance area covered by the electrodes $3 \mathrm{~mm} \times 3 \mathrm{~mm}$. In the case of $1 \mathrm{~cm}^{2}$ sensor the area covered by the electrodes was $6 \mathrm{~mm}$ $\mathrm{x} 6 \mathrm{~mm}$ while as the distance between the bond pads was $1 \mathrm{~cm}^{2}$. The last sets of sensors were over an area of $2 \mathrm{~cm}^{2}$ and the area covered by the electrodes was $1.2 \mathrm{~cm} \times 1.2 \mathrm{~cm}$. The electrodeelectrode distance for the $0.5 \mathrm{~cm}^{2}, 1 \mathrm{~cm}^{2}$ and the $2 \mathrm{~cm}^{2}$ was $0.5 \mathrm{~mm}, 1 \mathrm{~mm}$ and $2 \mathrm{~mm}$ respectively.

\section{Oxidation Step}

The wafer was placed in a Teflon cassette and was cleaned of all organics by placing the wafer in Piranha for 10 minutes at $70^{\circ} \mathrm{C}$. The Piranha solution was made by mixing $98 \%$ Sulfuric acid with $30 \%$ Hydrogen peroxide in a ratio of 9:1. The wafer was then quenched by dipping it in water 4 times. In order to remove the native oxide the cassette containing the wafer was submerged in BOE (Buffered Oxide Etch contains Hydrofluoric acid and $\mathrm{H}_{2} \mathrm{O}$ Transene) which was at $25^{\circ} \mathrm{C}$. The wafer was quenched by dipping the wafer in DI water 4 times and then it was put in a SRD (spin rinse and dry) machine. The next step was to grow an oxide layer which was done using a furnace. The predicted oxidation time for 500nm of oxide was done using the Deal-Grove equation: $t+\tau=\frac{X_{o x}^{2}}{B}+\frac{X_{o x}}{B / A}$ where $\mathrm{t}$ is required oxidation time, $\mathrm{X}_{\mathrm{ox}}$ is the required oxidation depth, $\tau$ is a time factor that accounts for any oxide present and it was 
estimated to be 0 and $\mathrm{B}$ and $\mathrm{A}$ are constants based on furnace temperature being used. The wafers were oxidized using wet oxidation in which initially the furnace was bought up to $900^{\circ} \mathrm{C}$. Thirty minutes into the furnace heating up the DI water was turned on and heated up to $105^{\circ} \mathrm{C}$. Low Purity Nitrogen was used to purge the furnace at 6 LPM; the wafer then was placed in a quartz boat with 2 dummy wafers on each side, and then placed in the center of the oven. The furnace temperature was increased to $1050^{\circ} \mathrm{C}$ and then placed in the oven for 90 minutes and the $\mathrm{N}_{2}$ was turned off. The furnace was turned off after 90 minutes and then left in the furnace overnight to cool down. The oxide was measured using Filmetrics F20, the oxidation layer was measured at the center of the wafer, and the right and left side of the wafer. The average thickness measured for the wafer was $3549.7 \AA$. The wafer was calibrated against a wafer with a known thickness of $8148 \AA$ and the device resulted in a value of $8137.5 \AA$.

\section{Sputtering}

Gold was sputtered on the wafer using a Denton IV Vacuum sputtering system. The wafer was placed onto the stage and system was bought down to $2 \mathrm{E}-5$ torr overnight by first turning on the roughing pump and then turning on the turbo molecular. High purity Argon was pumped into the sputtering system to bring the pressure up to $5.1 \mathrm{mtorr}$ and then rotation of the stage was started. The sputtering mechanism was then turned on and the sputtering was done at a rate of $5 \mathrm{~A} / \mathrm{sec}$ onto the wafer. The sputtering system was vented and the wafer was removed and then cleaned via Low Purity nitrogen. Once the wafer was cleaned the wafer was ready for the two part photolithography process in which the first part was using a positive photo resist to create the electrodes, the bond pads and the traces connecting the electrodes to the bond pads. 


\section{Positive Photoresist}

Shipley S1813 positive photoresist was used to create the electrode, the traces and the bond pads. $30 \mathrm{~mL}$ of resist was removed via a syringe from the refrigerator and placed in the photolithography room for 24 hours to bring it to room temperature. HMDS Primer 80/20 was also placed in syringe and a volume of $25 \mathrm{~mL}$ was extracted into the syringe. The gold sputtered wafer was centered on the Laurell Spin Coater using the centering tool and then applying a vacuum on the wafer. HMDS was dispensed on the center wafer until it covered the entire gold wafer. The spin coater was turned on and then spun at $300 \mathrm{rpm}$ at 30 seconds and then spun again for 20 seconds at 300 RPM. After the second spin cycle the rotation was stopped and $3 \mathrm{~mL}$ of the photo resist was poured on the wafer and special care was taken to avoid bubble formation during the pouring process. The spin coater was spun again where the spreading of the resist was done in two cycles the first one at $200 \mathrm{rpm}$ for $20 \mathrm{~s}$ and the second time at $500 \mathrm{rpm}$ for $10 \mathrm{~s}$. The next cycle was the planarization of the resist which was done at $4000 \mathrm{rpm}$ for $20 \mathrm{~s}$ and the last step was to slow down and stop the cycle which was done at $300 \mathrm{rpm}$ for $5 \mathrm{~s}$. The wafer was then soft backed at $90^{\circ} \mathrm{C}$ for $1 \mathrm{~min}$ and then chilled on a chiller plate for $10 \mathrm{~s}$.

\section{Lithography}

The photoaligner used was a Canon PLA501FA and the light integral for exposure time was done using a Light Dosage Meter (ILT1400). The mask was cleaned using $\mathrm{N}_{2}$ gas and DI water and was then cleaned with Isopropyl alcohol and the glass side was then cleaned using a lint-free wipe in order to minimized incorrect exposure due to lint residue. The optical head was moved and then placed back into position after the placement of the mask under the head. The mask was placed chrome side down until it was fitted in the mask holding area. In the case of the positive photoresist the positive photoresist mask was used and in the case of the SU-8 the negative photoresist masked was placed in the aligner. The Mask Load button on the aligner was 
pressed which resulted in the vacuum being turned on and fixing the mask in its place. The mercury lamp vapor lamp was turned on and allowed to warm for 30 minutes. The silicon wafer with the photoresist was then placed on the onto 2 rubber band tracks until the sensors picked up the wafer and placed it $30 \mu \mathrm{m}$ under the mask. The light exposure time was set by fixing the light integral on the dials to 4 for the positive photoresist. Once the exposure time had elapsed the aligner placed the wafer in a wafer cassette, which led to the development of the exposed photoresist.

The exposed wafer was placed in a Shipley S1813 Developer solution for 10 minutes to create traces and electrodes and prepare the wafer for the wet etching process. After development the wafer was placed in the gold etchant for 12 minutes to remove all the excess gold. After the gold was etched away leaving only the electrode, the traces and the bond pads. The polymerized Shipley S1813 positive photoresist was stripped using acetone. The wafer with the positive photoresist was placed in a solution of acetone for 15 minutes while being gently agitated. After 15 minutes the wafer was examined under the Brightfield microscope to make sure no residues were remaining, if any residues were found the wafer was placed in acetone for another 2 minutes and then the wafer was inspected again. Once no traces of the photoresist were found the wafer was then moved onto the next step of placing a negative photoresist on the wafer.

\section{Negative photoresist}

SU-8 negative photoresist was used to create an electrical insulator so that the traces would not act as electrodes thereby reducing the resolution for impedance spectroscopy. The wafer was placed in the center of the spin coater using a centering tool and then the vacuum was turned on in order to hold the wafer in place. HMDS primer was not used in the SU-8 process. The SU-8 was placed was placed on the center of wafer and then spun at $3000 \mathrm{rpm}$. The wafer 
was then aligned using a similar photoaligner step with an integration time of 37 instead of 4 which was present in the case of the positive photoresist. The photoresist was then placed in the developer solution for 15 minutes to just expose the electrodes and the bond pads and cover the trace wires. The wafer was inspected under a bright field microscope to make sure that the electrodes and the wafers were properly exposed. If residue of the negative photoresist was found on the electrodes or the wafer the wafer was placed in the developer solution for another 10 minutes. Once the wafer passed the optical inspection it was then rinsed with Isopropyl alcohol and then rinsed with DI water and air dried using low purity $\mathrm{N}_{2}$ gas. Once all the photoresist on the electrodes and bond pads were removed then the entire wafer was inspected for any incomplete connection between the bond pads and the electrodes. If any incomplete connections were found those connections were then noted and were not used for experimentation.

\section{$\underline{\text { Experimental Set-up }}$}

\section{Raw And Cooked Chicken}

Melanoma cells have different water content as compared to their healthy skin analogue. An analogue was needed as a proof of concept to show that the electrodes could pick up the water content between two tissues. Since there is a large difference in water content between cooked and raw chicken, the electrodes were tested with raw chicken and cooked chicken. The ability of the sensor's ability to pick up impedance difference between raw and cooked chicken would be an indicator of the differentiating ability of the sensor to pick up tissue with varying tissue moisture content. The raw chicken was a Foster Farms chicken bought from the local grocery store. The skin and muscle together were compared of the raw chicken and cooked chicken and the muscle of the raw chicken was compared with muscle of the cooked chicken. In 
both cases the chicken was cooked for a period of 40 seconds in a regular household microwave.

The chicken samples in this thesis were cut into pieces smaller than $1 \mathrm{~cm} \times 1 \mathrm{~cm}$ (prior to cooking) in order to place the chicken only on the electrodes.

\section{Electrical Connectivity And Testing Protocol}

In order to validate that the electrodes could measure impedances accurately, resistors were placed across a few sensors. This was accomplished by using micromanipulators. The probes were then touched down onto the bond pads and the electrodes. The connectivity between the electrode and corresponding bond pad was established by using an Agilent digital multimeter(DMM). Once electrical connectivity was established a resistor was placed between 2 electrodes and the impedance was measured from the bond pads. The resistances were measured over three different types of sensors in order to prove that that all complete connections were working properly. The same resistors were used in-order to minimize tolerance variations between the resistors, a precise resistance value of the resistors were taken by measuring the impedance of the resistor using the DMM. The DMM value was then compared against the value measured by the electrodes. All electrodes with diameters of $50 \mu \mathrm{m}$ were not used due to the fact that the SU-8 developer solution was not able to completely expose the $50 \mu \mathrm{m}$ diameter electrode making it difficult to touchdown the probe onto the gold. The sensors that were tested on were the $2 \mathrm{~cm}^{2}$ sensor with an electrode diameter of $250 \mu \mathrm{m}$ and the $2 \mathrm{~cm}^{2}$ and $1 \mathrm{~cm}^{2}$ sensor with an electrode diameter of $100 \mu \mathrm{m}$. The electrodes used for this testing purpose were not used again in order to avoid high resistance values caused by repeated touchdowns which would result in the gold bond pad being scratched of leaving on the silicon dioxide layer, thus decreasing conductance. The sensors used have been labeled as R1-R3 and location on the wafer has been shown through the usage of the mask image (See Figure 10). 
Table 1. Resistance between electrodes

\begin{tabular}{|c|c|c|c|c|c|c|}
\hline Name & $\begin{array}{l}\text { Sensor } \\
\operatorname{Size}\left(\mathrm{cm}^{2}\right)\end{array}$ & $\begin{array}{l}\text { Electrode } \\
\text { Diameter }(\mu \mathrm{m})\end{array}$ & $\begin{array}{l}10 \mathrm{~K} \Omega \\
\text { resistor } \\
\text { value } \\
\text { measured }\end{array}$ & $\begin{array}{l}46 \mathrm{~K} \Omega \\
\text { resistor } \\
\text { value } \\
\text { measured }\end{array}$ & $\begin{array}{l}\text { Percent } \\
\text { Error for } \\
10 \mathrm{~K} \\
\text { compared } \\
\text { to } 10 \mathrm{~K} \\
\text { control }\end{array}$ & $\begin{array}{l}\text { Percent } \\
\text { Error for } \\
46 \mathrm{~K} \\
\text { compared } \\
\text { to } 46 \mathrm{~K} \\
\text { control }\end{array}$ \\
\hline $\begin{array}{l}10 \mathrm{~K} \\
\text { resistor } \\
\text { control }\end{array}$ & - & - & $9.8576 \mathrm{~K} \Omega$ & - & - & - \\
\hline $\begin{array}{l}46 \mathrm{~K} \\
\text { resistor } \\
\text { control }\end{array}$ & - & - & - & $46.689 \mathrm{~K} \Omega$ & - & - \\
\hline R1 & 2 & 250 & $9.8879 \mathrm{~K} \Omega$ & $\begin{array}{l}46.723 \mathrm{~K} \\
\Omega\end{array}$ & $.304 \%$ & $.073 \%$ \\
\hline $\mathrm{R} 2$ & 2 & 100 & - & $\begin{array}{l}46.728 \mathrm{~K} \\
\Omega\end{array}$ & - & $.084 \%$ \\
\hline R3 & 1 & 100 & $\begin{array}{l}9.8809 \mathrm{~K} \\
\Omega\end{array}$ & $\begin{array}{l}46.707 \mathrm{~K} \\
\Omega\end{array}$ & $.236 \%$ & $.039 \%$ \\
\hline
\end{tabular}

Table 1 shows how much resistance the MEA traces add when measuring an electrode. This

shows also the amount of error is associated with the two resistances. The greater the resistance

the lower the resistive effect of the electrodes as the resistance of the object being measured becomes greater and greater. This lowering effect is seen in the $\%$ error columns for the two resistors. Once it was shown that resistance values could be accurately measured for the sensors all the remaining sensors that passed the optical inspection were deemed fit for measurement with tissue. The next step was to test how well the sensors could pick up impedance measurement between cooked chicken and raw chicken. Since impedance data was needed, the DMM used for connectivity validation would not suffice and an automated acquisition method was need. This was accomplished by using NI-ELVIS prototyping board and LabView. 


\section{NI-ELVIS And LabView}

NI-ELVIS (National Instruments Educational Laboratory Virtual Instrumentation Suite) is a prototype board with various testing components. The NI-ELVIS has a variable voltage generator, an oscilloscope, a function generator, an impedance analyzer, and a DAQ (Data Acquisition device) to communicate with the computer. The impedance analyzer was tested using a $0.1 \mu \mathrm{F}$ capacitor in parallel to a $3 \mathrm{~K} \Omega$ resistors and capacitor values of $1 \mu \mathrm{F}$, and $10 \mathrm{nF}$ were used and resistor values of $1,10,100 \mathrm{k} \Omega$ were measured in the impedance analyzer at different frequencies in order to see how well the analyzer measured the impedance. The system was automated using LabView. LabView is a graphical programming environment that interfaces with NI-ELVIS and various measurement devices on the NI-ELVIS board and was used for data acquisition purposes in this thesis.

In order to measure the impedance on chicken the built in impedance analyzer was used and measurement was taken after passing the current through the chicken for 10 seconds. The impedance analyzer measured the impedance at $2 \mathrm{kHz}$ and at $10 \mathrm{kHz}$ for experiment 2 and at the frequencies showed in Table 1. The measurements were taken for the first experiment only across two electrodes and are labeled as T1S in the appendix. Each frequency for each piece of chicken had 2 replicates. A total of 7 raw chicken pieces were used for this experiment. 


\section{Experiments}

\section{Creating The MEA}

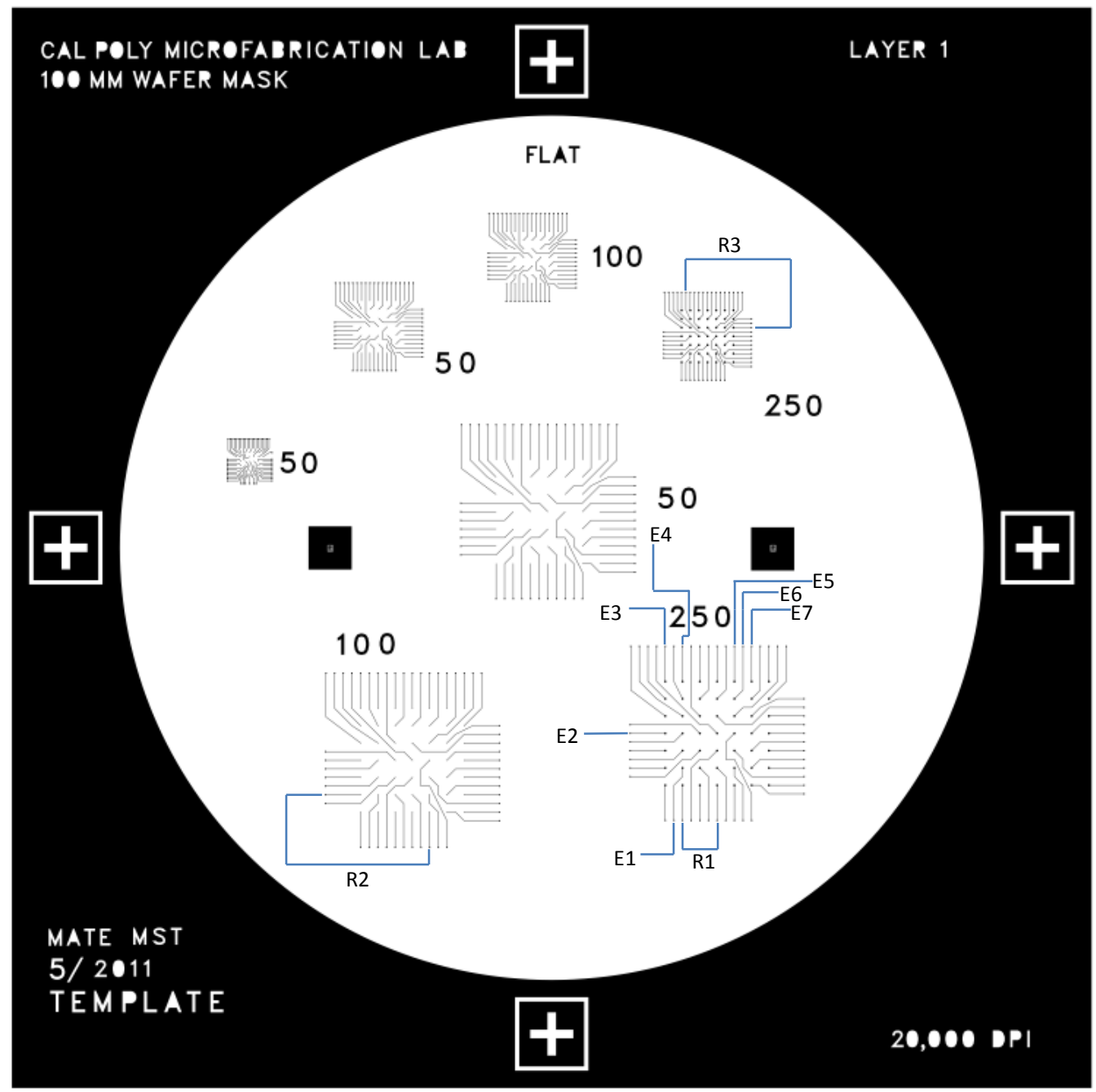

Figure 10. Positive photoresist mask used for MEA with electrodes used in experiments 


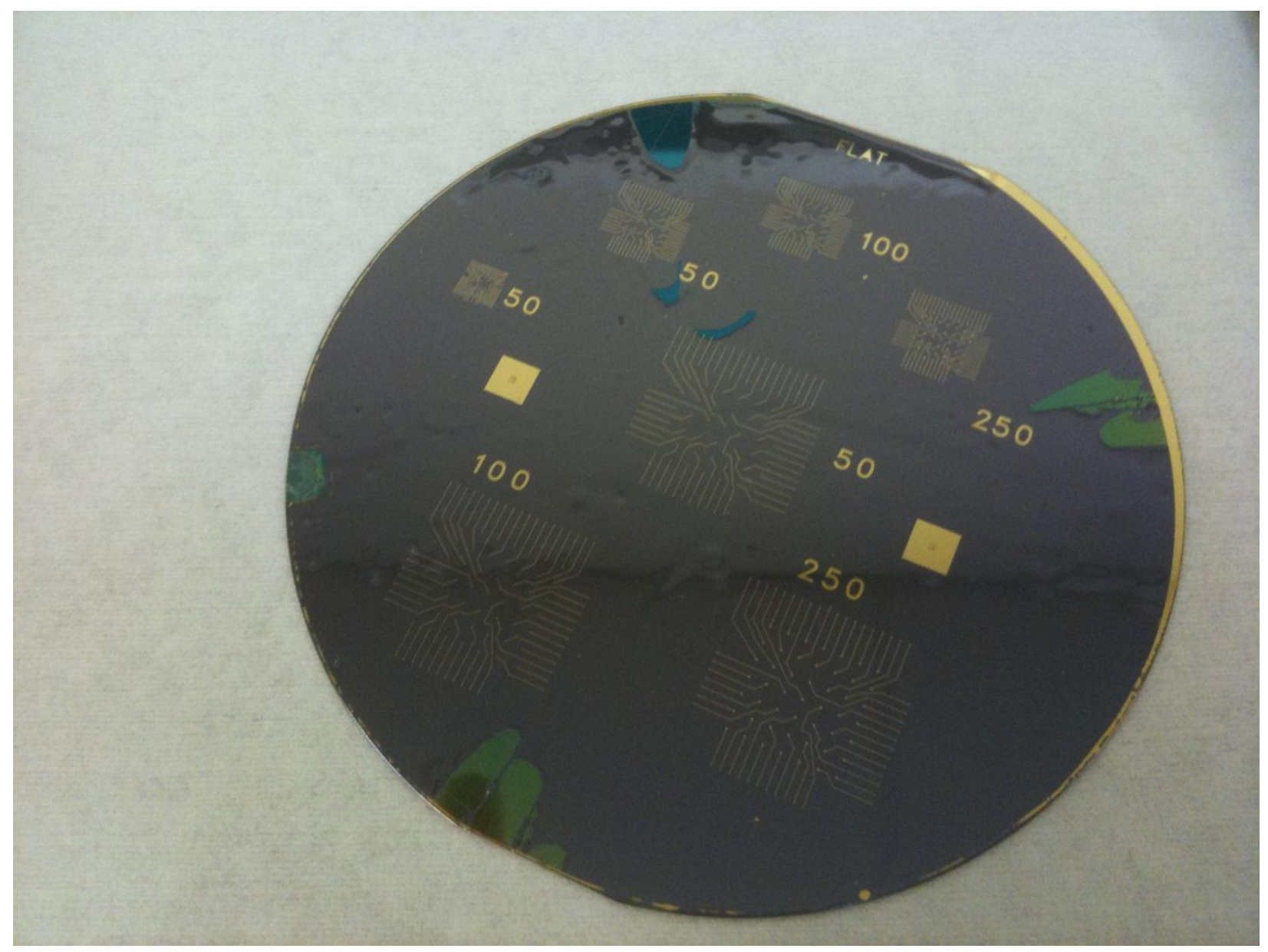

Figure 11. Finished MEA after fabrication process.

The MEA was made using a mask as shown in Figure 10. The black lines inside the white circle represent how the gold traces would be formed. The mask was used for exposing the positive photoresist where all the areas that are "white" would be dissolve away during the development and the black traces would cross link, creating a protective layer during the etching process. Figure 11 shows how MEA looks after the microfabrication process has been completed. The electrodes were the circular ends and at the other end was where the microprober made electrical contact with the bond pad. Figure 10 also shows the locations where measurements were taken from. 


\section{Experiment I}

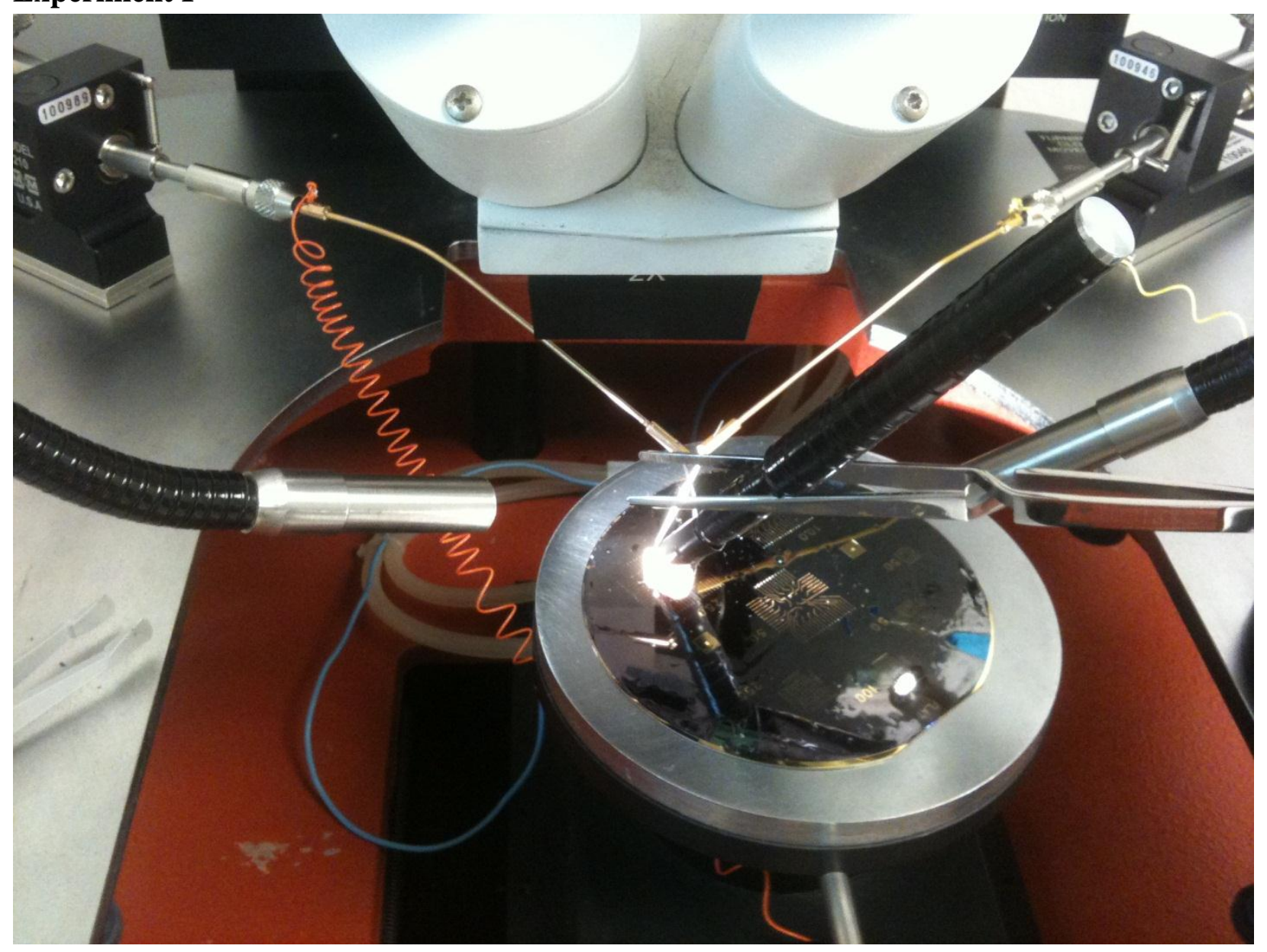

Figure 12. Chicken on microelectrode with weight on the chicken while being microprobed. The first experiment used 7 pieces of raw and cooked chicken. In these experiments the chicken samples contained skin, and it was the skin that came in contact with the sensors (as shown in Figure 12). The chicken samples were measured in two different ways. Since the electrodes were recessed below the photoresist layer the impedance was measure with an nonconductive weighted object that would weigh the chicken onto the electrode and another one without out any weighting system. The weighting system was employed in order to see if the recessed electrodes would create an impedance difference for chicken that was weighted down and chicken that wasn't. The $2 \mathrm{~cm}^{2}$ sensor with containing electrodes with a diameter of $250 \mu \mathrm{m}$ was used and was labeled as T1S (Figure 10). The measurement was taken using the NI-ELVIS 
and LabView. The LabView program (as shown in Figure 13) took a measurement after allowing the current to pass through the chicken for 15 seconds, and it took 2 replicate measurements of the chicken at frequencies of $0,2.5,5,7.5,10,25,50,75,100,250,500,750$, 1000, 2500, 5000, 7500, and 10000Hz. The impedance measurements were taken by touching the micromanipulators to the bond pads which were connected to the "Current HI" port and another connected to the "Current LO" port. The reported values were placed in a text files and then later transported to Excel.

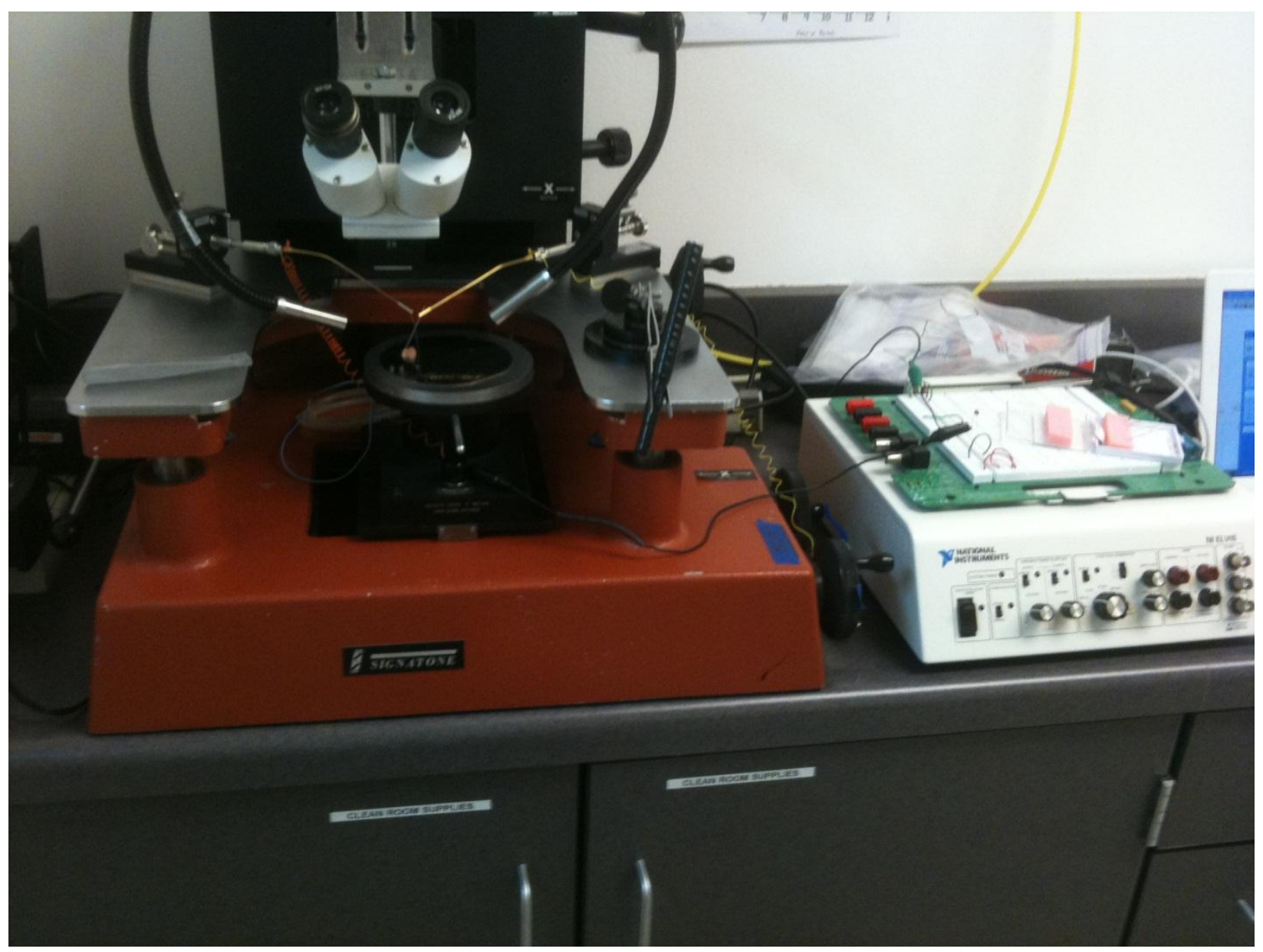

Figure 13. Experimental set-up with microprobe, NI-ELVIS, probe station and chicken for measuring impedance. 


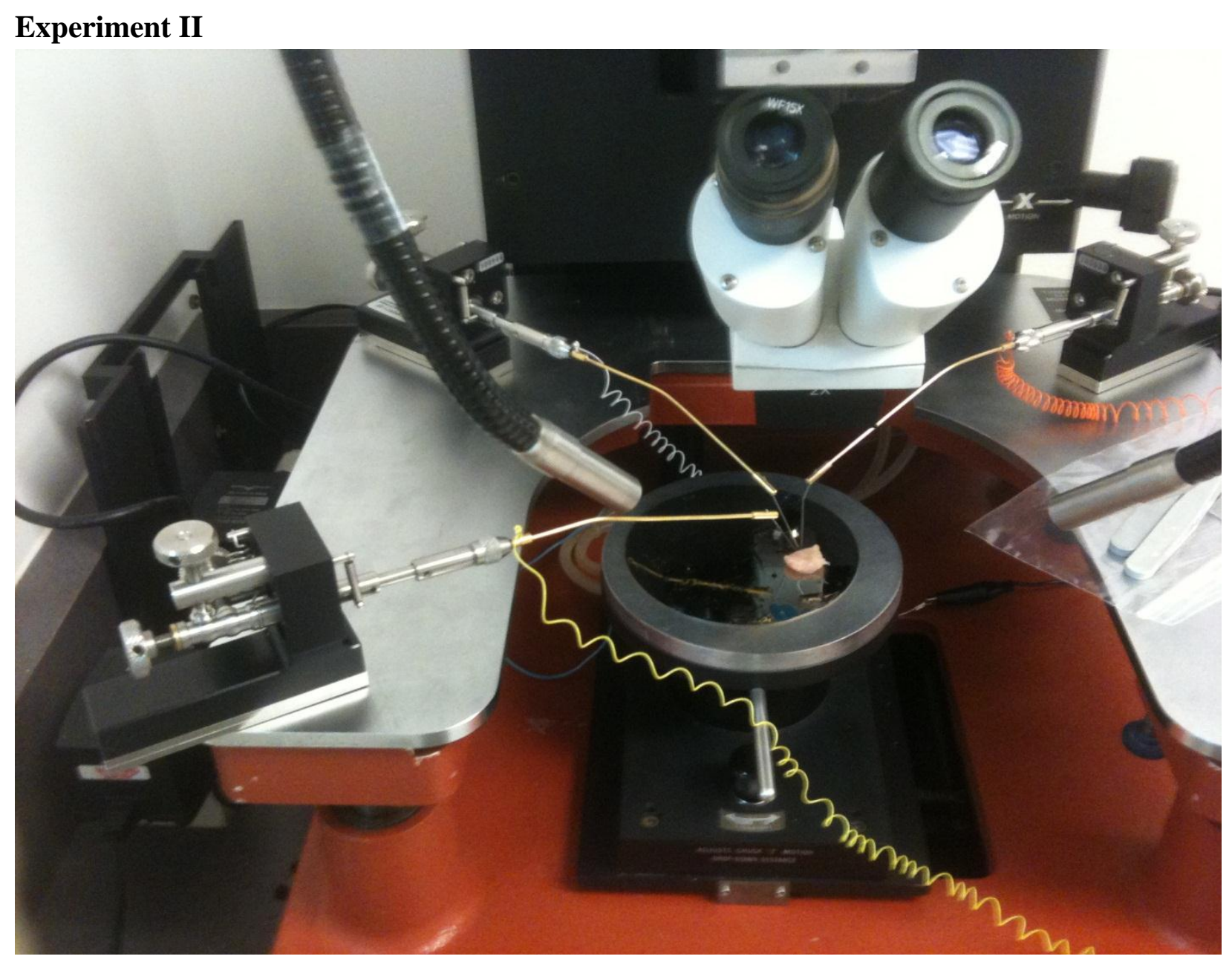

Figure 14. Three electrode set-up used in Experiment II by using three probers and the impedance measured between three electrodes.

This experiment didn't use the weight system but measured the impedance across 3

electrodes (Figure 14). There were 13 samples of raw and cooked chicken that were measured.

Since the analyzer could only measure the impedance between two electrodes at a time, the micromanipulators connected to the impedance analyzer were iteratively changed in order to map the impedance in all three orientations. In this experiment the $2 \mathrm{~cm}^{2}$ sensor with a $250 \mu \mathrm{m}$ diameter electrode was used. The samples were measured at frequencies of $2 \mathrm{kHz}$ and $10 \mathrm{kHz}$. Each instance of the frequency and electrode orientation was measured in 5 replicates in order to get an average impedance value and to see the spread of the impedance after repeated 
measurements. The electrodes were $1.875 \mathrm{~mm}$ apart and $4 \mathrm{~mm}$ apart to see how the interelectrode difference affects the impedance being measured. As in Experiment I the current was allowed to flow between the two electrodes for 10 seconds in order to allow for electrical relaxation of the chicken. The data was written on to a text file which was later transported to excel. Once the experiment was completed the wafer was first washed with ethanol, then with DI water and then placed in a spin rinse drier to clean the wafers.

\section{Experiment III}

The last experiment measured the impedance across two electrodes at $2 \mathrm{kHz}$ with an inter electrode difference of $4 \mathrm{~mm}$ between the two electrodes. Ten chicken samples were used where five were cooked chicken and five were raw chicken. The impedance was measured across the electrodes via NI-ELVIS (LabView Program shown in Figure 21). This experiment was done due to the results of experiment II. 


\section{Results:}

Each time a measurement was taken, new electrodes were used in order to avoid repeated scratching of the gold sputtered bond pads which could result in increased resistance thus convolving the impedance measurements.

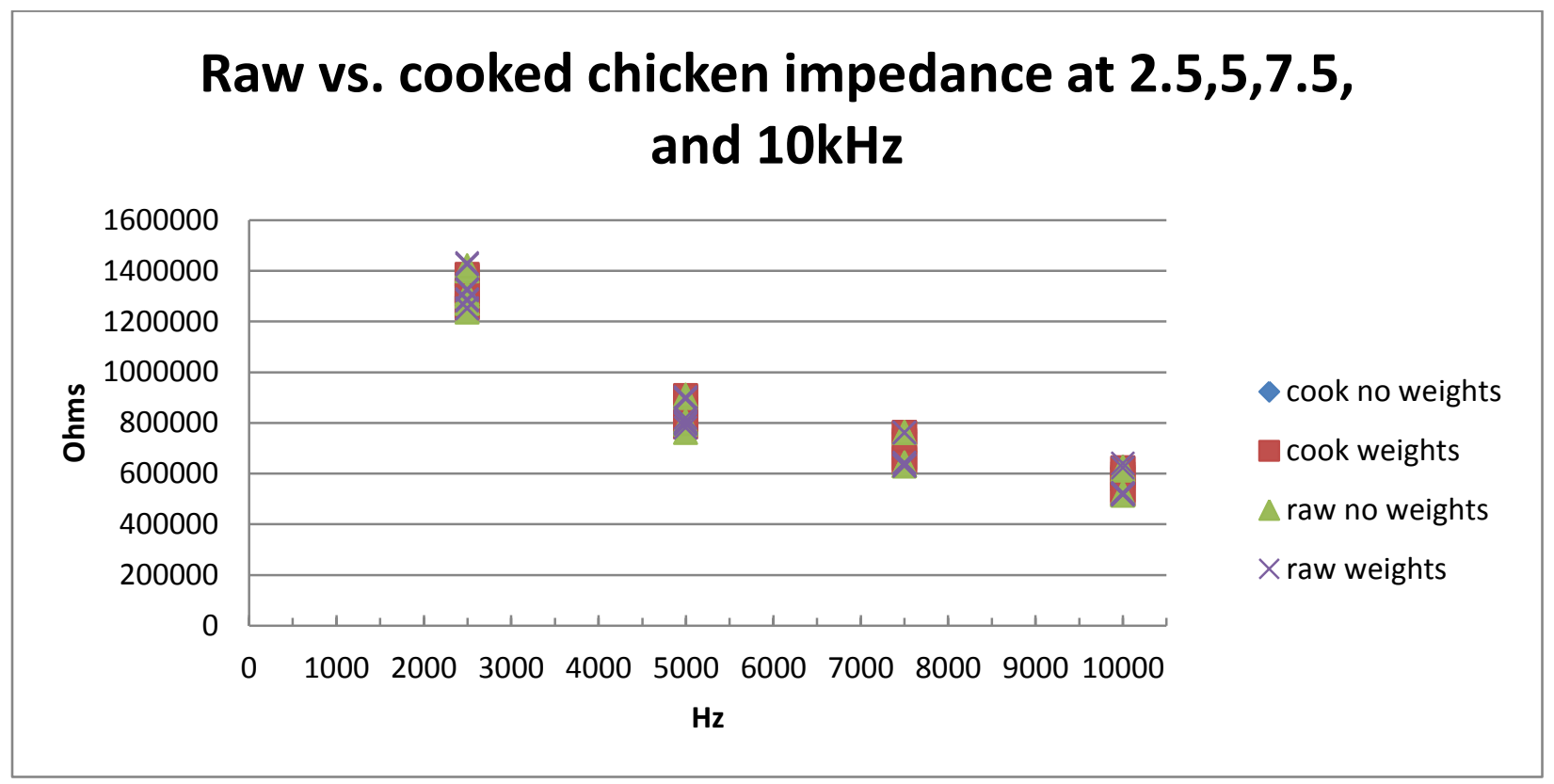

Figure 15. Impedance measurement taken of the raw and cooked chicken (with and without weights) data over 2500, 5000, 7500, 10000Hz. Electrodes E1 and E2 used (as shown in Figure 10).

Figure 15 shows the impedance of the raw chicken and cooked chicken with and without weights. The weights were added under the assumption that the recessed electrodes would not make proper contact with the chicken. This experiment showed if the addition of weights affected the impedance values and if the electrodes could detect the difference between raw and cooked chicken at various frequencies. 


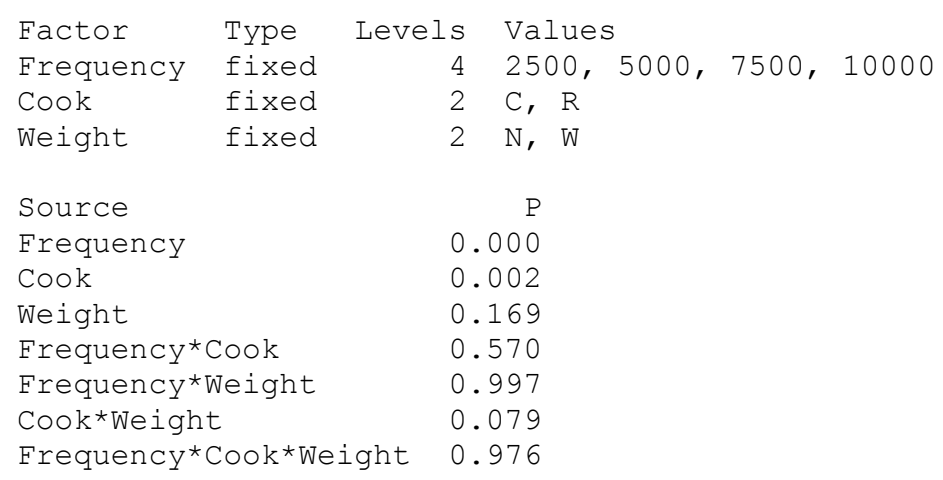

Figure 16. General Linear Model: Impedance versus Frequency, Cook, Weight. Figure 16 shows a summary of the general linear model in which a Tukey test was performed, it showed the interactions between the weight on the chicken, measurement frequency, and temperature (cooked vs. raw). The Tukey test showed that the chicken temperature and the measurement frequency both independently affected the impedance of chicken being measured. This method was used to show the complex interactions occurring between 3 variables that affect the response variable which is impedance. The p-value for frequency and for cook was less than the .05 showing that there was statistical effect by frequency and chicken temperature on the impedance. The non-overlapping regions were also only seen for Frequency and "cook" (cooked vs. raw), indicating that frequency and the cooked vs. raw chicken effects the impedance of the chicken independently (As shown in Appendix). The interaction between frequency and cook (cooked vs. raw) does not affect the impedance of the chicken. However there is not enough statistical evidence to show that there was an impedance difference caused by interaction of the weighing the chicken down, the measurement frequency and if the chicken is cooked or raw. 


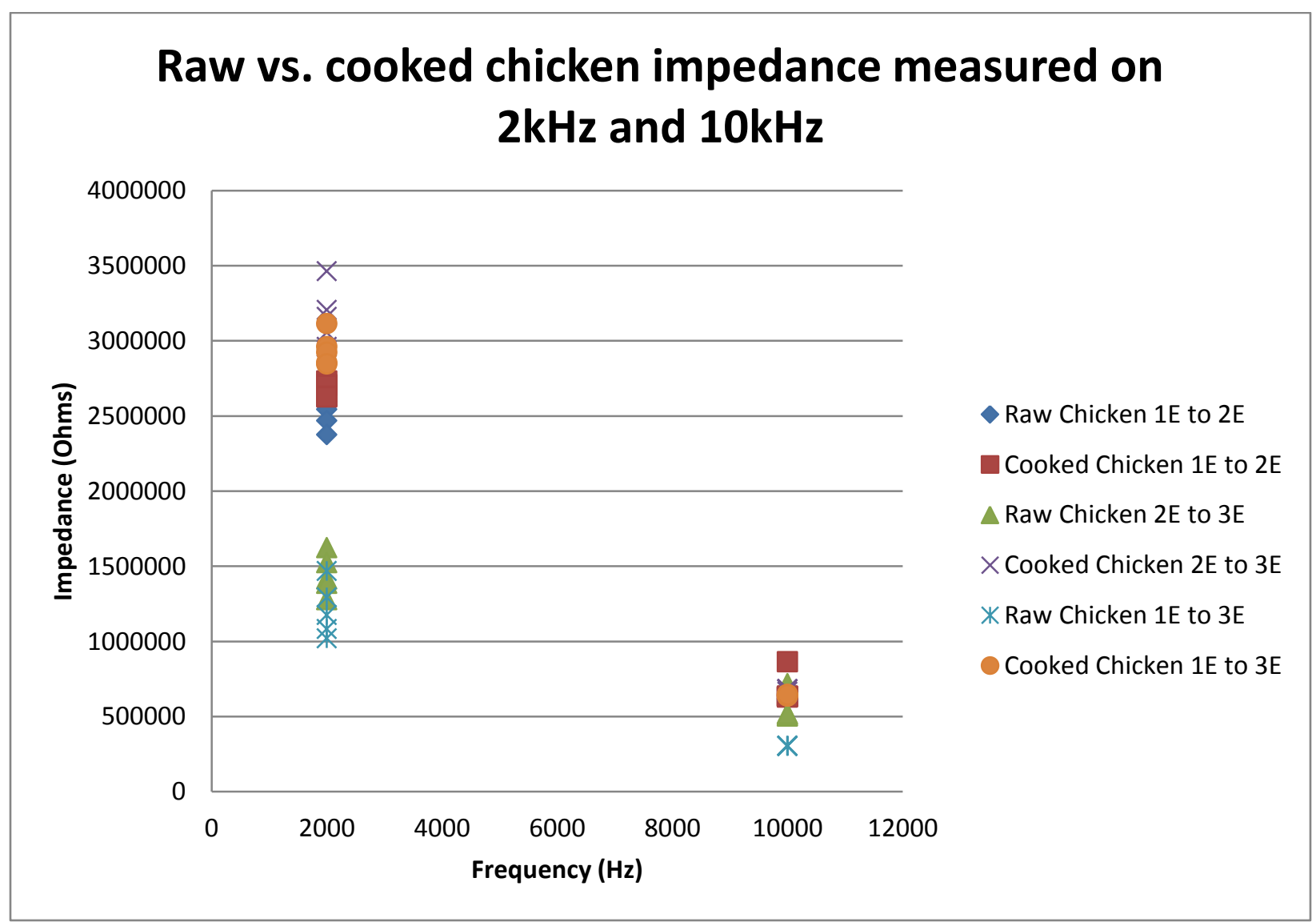

Figure 17. Impedance measurement raw vs. cooked chicken with 3 electrodes but only 2 electrodes were used at a time. (1E-E5, 2E=E6, 3E=E7).

The graph above (Figure 17) shows the raw impedance measurement of cooked vs. raw chicken at measurement frequencies of $2 \mathrm{kHz}$ and $10 \mathrm{kHz}$. The electrodes used in these measurements are labeled as electrodes E5-E7 (as shown in the Figure 10). 


\section{Electrode by Frequency LSMeans Differences Tukey HSD \\ $\alpha=0.050$ \\ LSMean[i] By LSMean[j]}

\begin{tabular}{|l|l|l|l|r|}
\hline Level & & & & Least Sq Mean \\
\hline$A, 2000$ & $A$ & & & 2571221.5 \\
\hline$B, 2000$ & A & & & 2416839.9 \\
\hline $\mathrm{C}, 2000$ & & $B$ & & 1651202.0 \\
\hline$A, 10000$ & & & $C$ & 618550.5 \\
\hline$B, 10000$ & & & $C$ & 593264.0 \\
\hline$C, 10000$ & & & $C$ & 396127.5 \\
\hline
\end{tabular}

Levels not connected by same letter are significantly different.

Figure 18. R-Output summary of a Tukey HSD test that looks at statistical differences in frequencies applied between various electrodes.

\section{Electrode by Temp \\ LSMeans Differences Tukey HSD \\ $\alpha=0.050$ \\ LSMean[i] By LSMean[j]}

\begin{tabular}{|l|l|l|l|r|}
\hline Level & & & & Least Sq Mean \\
\hline A,C & A & & & 1721478.5 \\
\hline B,C & A & B & & 1621379.1 \\
\hline A,R & A & B & & 1468293.5 \\
\hline B,R & A & B & 1388724.8 \\
\hline C,C & & B & & 1382542.2 \\
\hline C,R & & & C & 664787.3 \\
\hline
\end{tabular}

Levels not connected by same letter are significantly different.

Figure 19. R-Output summary of a Tukey HSD test that shows the stastical differences in raw vs. cooked chicken ( $\mathrm{R}$ is raw and $\mathrm{C}$ is cooked), the variable is represented by temperature, and its impedance measurement across various electrodes.

Figure 18 and 19 showed that there was a significant difference in raw vs cooked chicken impedance for electrodes that were $4 \mathrm{~mm}$ apart (Electrodes E5 and E7) with a measurement frequency of $2 \mathrm{kHz}$. The variable temperature indicates the two states cooked and raw. The method was based on the least means squares using the Tukey method where non overlapping letters indicate statistical difference between the two categories. 


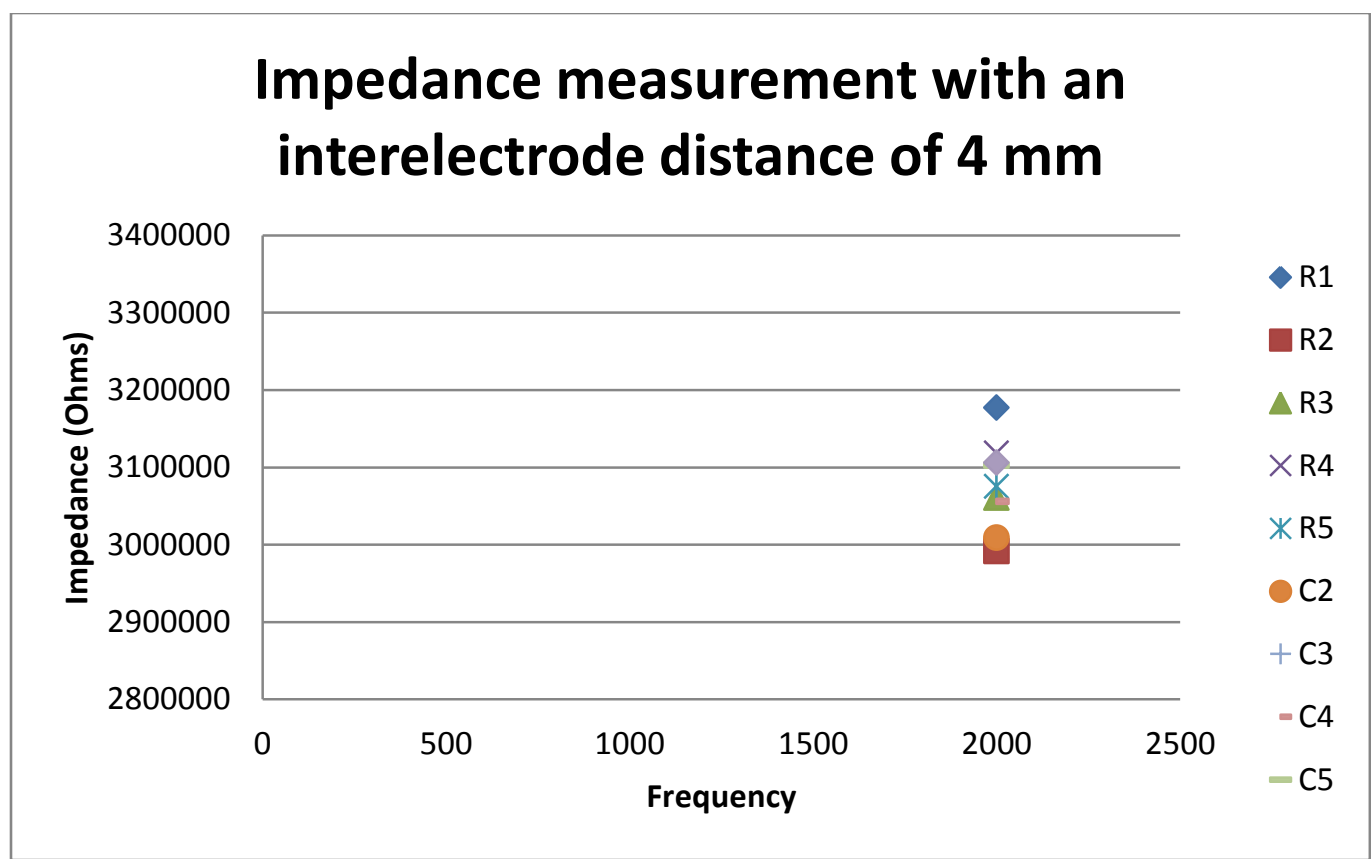

Figure 20. Impedance measurement of raw vs. cooked chicken with an inter-electrode distance of $4 \mathrm{~mm}$ measured at $2 \mathrm{kHz}$.

Cooked vs. raw measurements were taken from 2 electrodes with an inter-electrode

difference of $4 \mathrm{~mm}$ between the two electrodes. No statistically significant differences were seen between the impedance of cooked vs. raw chicken (Graph showed in Figure 20). The electrodes used were electrodes E3 and E4 (Figure 10).

Two-Sample T-Test and Cl: R, C

\begin{tabular}{lrrrr}
\multicolumn{4}{l}{ Two-sample $T$ for $R$ vs $C$} \\
\multicolumn{1}{c}{ N } & Mean & StDev & SE Mean \\
R & 5 & 3084686 & 68937 & 30830 \\
C & 5 & 3048928 & 58843 & 26315
\end{tabular}

Difference $=m u(R)-m u(C)$

Estimate for difference: 35758

95\% CI for difference: (-60089, 131604)

$\mathrm{T}$-Test of difference $=0$ (vs not $=$ ) $:$ T-Value $=0.88 \quad$ P-Value $=0.407 \quad \mathrm{DF}=7$

Figure 21. Minitab output of the t-test result for raw chicken (R) vs. cooked chicken impedance (C), at a measurement frequency of $2 \mathrm{kHz}$. 
A p-value of 0.407 supports the null hypothesis that the impedance difference between raw and cooked chicken at $2 \mathrm{kHz}$ is indistinguishable with $95 \%$ confidence. Figure 20 showed that at a second attempt the difference in raw vs. cooked chicken could not be seen.

\section{$\underline{\text { Discussion }}$}

\section{MEA Wafer Validation}

Using a similar concept as State and Fleshmen et al. [12,13]. Resistors were placed between the electrodes to see how accurately the MEA was able to measure resistances across the electrode. The electrodes that were used had to pass a visual inspection. The inspection involved visually seeing that the traces were connected from the electrode to the bond pad. Any electrodes with traces that were not connected properly, were not used in the experimentation process. The difference between the actual value of the resistor vs. the measured value of the resistor using the Agilent digital multi-meter. It was assumed that if measurements were made across 3 sensors, the rest of the sensors that had passed visual inspection were also considered as usable electrodes. The data showed that the electrodes measured the resistance with a mean \%error of $.064 \%$ for $46 \mathrm{k}$ ohm resistor and $0.272 \%$ for $10 \mathrm{k}$ ohm resistor. The possible reason for the different in percent error was due to the fact that the resistance by the gold trace was proportionally a larger quantity of the resistance reading as compared to the $46 \mathrm{k} \mathrm{ohm}$. No capacitance was done, but it was assumed that since the resistance reading had very low percent error, and that the resistance readings proved connectivity and were enough proof that the MEA could accurately measure the resistance across the electrode. This was used for consequent measurement steps in the experimentation process. In future experiments a capacitor should be placed between the electrodes and measure how well the system can measure capacitance. The control was done using a DC signal but the measurements were taken with an AC signal. The 
DC baseline was characterized but the AC baseline was not characterized. The baseline for AC could have been very different from the DC case. One of those reasons could be the fact that the curling of the wire could have added impedance to the measurement which could have affected the data being measured. When the lines are twisted up as shown in Figure 13 and 14, inductance could have played a role in the impedance measurement. That being the case it could be possible that impedance measurement taken from the tissue included the inductance measurement which could have convoluted the data. Having an AC control would also give an indication of baseline, if the measurements were expected to be below the baseline, the signal could not extracted. The other control could be to use PDMS as a calibration standard before testing with chicken. This is important since chicken is an inhomogeneous and anisotropic material, the orientation would affect the impedance. PDMS could provide a material that is homogeneous and isotropic and this could be used as a reference to see the variation in impedance across different electrode lengths. The variations in impedance would then be caused not by impedance but inherent different in the MEA, which would give the state of each electrode, before taking the chicken measurement.

Since extra precaution was not taken to oxidize the unpolished side of the wafer, it could be possible that the unpolished side would not have completely oxidized and doped silicon could be exposed. Doped silicon is a great electrical conductor and could have also effected the measurements. In order to guarantee that the doped silicon from the unpolished side is indeed behaving as an insulator, a conduction measurement should be taken in order to prove that the oxide layer is thick enough to provide electrical isolation. Having no oxide on the unpolished side could allow current to pass through the probe station and into the wafer which would finally affect the impedance reading. 


\section{Raw Chicken vs. Cooked Chicken}

The results showed that the MEA could potentially distinguish between cooked and raw chicken, but there was a strong indication that a better measurement method and cleaning protocol could increase the sensor's ability to distinguish the impedance levels. The tests indicated with a p-value of less than .05 that the sensors picked up a difference in impedance levels between cooked and raw chicken at a frequency of $2000 \mathrm{~Hz}$. The third experiment was ran to see if the experiment was repeatable, but it seems that the experiment could not be replicated as indicated by the Minitab output (Figure 20) in the results section. The reason for the nonreplicability of the test could be due to the fact that after the first run, the oils present in the chicken and chicken tissue would adhere to the SU-8 and to the gold electrode surface. This could have created a problem because it could act as an additional capacitor and/or resistor, thereby skewing the data for subsequent measurements. The cleaning protocol to remove chicken residue was to clean the MEA with ethanol, but this method did not seem effective. It would cause the tissue to become dehydrated and adhere more strongly to the MEA even after spin rinse and dry to clean the wafer. A process involving Tween-20 might be much more effective due to its ability to act as a non-polar solvent to dissolve the oils present on the electrode. Measurements taken at frequencies higher than $2 \mathrm{kHz}$ caused the impedances to decrease as seen in Figure 10. The higher frequency could be causing a frequency dependent capacitive response of the chicken tissue for both cooked and raw chicken. In one experiment at $2 \mathrm{kHz}$ it was seen that there was a difference which could be attributed to possible difference in water content because the only parameter that was changed was cooking the chicken. Cooked chicken had an higher impedance as compared to raw chicken. The difference was seen when the electrode was placed $4 \mathrm{~mm}$ away. The measurement was a differential measurement as compared to using a larger reference electrode. A reference electrode would allow 
measurements to be taken from a known reference, allowing for the difference to be measured with better accuracy as compared to the differential measurement. Since the electrodes didn't have precise measurement potential to be measured from, there was no way to normalize the two electrodes. Although, a differential measurement might be able to remove those offsets caused by the electrodes, but there would be no way to get rid of the gain effect caused by the electrodes. The double layer of the electrodes could be different for both electrodes which could affect the overall impedance being measured. At higher frequencies the double layer capacitance at sensors could get shorted resulting in bypassing the resistor in parallel (as modeled in a perfect $\mathrm{RC}$ electrode). The electrochemical solution to create the electrical current were the ions that were present in the raw chicken and the fluid present in the chicken allowed for free movement of ions resulting in lower impedance. When the chicken was cooked the impedance changed due to the fact that the movement of ions through solution became more difficult creating a higher impedance in terms of capacitance and resistance because it was harder for current to move through the solution. The lower presence of the solution on the electrodes limits the exchange of ions via the electrode. Gold electrodes are also polarizable which results in the fact that when a nonreactive metal (such as gold) is in equilibrium with the electrolyte solution, the electrode interface builds up charge like a capacitor and does not cause current to flow through (even leakage current). Metal such as Silver allow for exchange of ions between $\mathrm{Ag}^{+}$and $\mathrm{Cl}^{-}$and the metal is non-polarizable so charge does not build up as easily, since the chlorine can keep exchanging ions with silver creating a current. Changing the potential applied on the $\mathrm{AgCl}$ can reverse this effect and then revert the ionic complex back to pure silver. Another possible metal could be platinum which has known to have the similar properties as silver in terms of impedance spectroscopy. Electrodes that were not recessed could have also increased signal 
fidelity because the electrodes could act as needles and pierce the tissue allowing for exposure to greater amounts of solution hence allowing greater exchange of current. It would allow for electrochemistry using the solution that is present in the tissue instead of having to use a nonnative solution. Another way to improve signal impedance would be to use a more precise impedance analyzer method. NI-ELVIS impedance analyzer can cause averaging of the impedance values resulting in lower precision of the values which can tend to again skew the impedance values to below statistical values. Another method could be using a shunt resistor. A shunt resistor would be placed in series between the two electrodes so that the voltage would be applied to one electrode the current would pass through the electrode, to the tissue then through the shunt resistor ending at the other electrode which would be grounded. The shunt resistor would be a resistor of a known value with very low tolerance, and the voltage drop across resistor would be measured (the waveform of the current would be taken in). A scope such as those provided by Tektronix could be connected to the computer so that data of the current waveform could be written into the computer. Simultaneously the voltage waveform would also be measured using another scope and placed into the computer. The voltage would be divided by the current to give a measure of the shift in phase which would give information about the capacitance and the change in amplitude would give information about the resistance through the tissue. The shunt resistor would have to be much smaller than the impedance value being measured, usually a value of $1 \mathrm{ohm}$ is used as a shunt resistor with very small tolerance to give a high precision current value.

Electrodes of diameter 250um were only used to measure the impedance across the chicken. The 100um and 50um diameter electrodes were not used because the SU-8 developer was not able to successfully expose all the electrodes. This left a majority of the electrodes 
covered by the SU8 making impedance measurement of the chicken impossible via the smaller diameter electrodes.

To avoid issues with constantly scratching off the surface of the bond pads, a sacrificial layer of titanium could be sputtered on and then a gold layer on top of the titanium. This would allow for better adhesion of the metals to $\mathrm{SiO}_{2}$ since gold has poor bonding qualities with $\mathrm{SiO}_{2}$. The titanium layer would also allow for repeated touchdowns due to the reduced scratching of the bond pads from the probe pins. This would allow for the same sensor to be used multiple times in multiple experiments. The bond pads were 100um by 100um square, which only allowed one time use of the electrodes, in order to reduce effect of repeated scratching. The bond pads that would be designed to get around this constraint would be to use $5 \mathrm{~mm}$ by $5 \mathrm{~mm}$ bond pads. These bond pads could be connected to the circuit by placing conductive epoxy on the bond pads and connecting wires to the actual circuit. This would have allowed for the electrodes to be reused over time, but then the problem of polarizable electrodes would become more apparent, where the metals discussed in the discussion section would become useful.

The electrodes that were used were only $250 \mathrm{um}$ electrodes due to the fact that the SU-8 developer could not successfully develop the 100um and 50um electrodes. This meant that electrical contact could not be made with the smaller electrodes which was also due to limits of the photolithography system being used. In the future the $250 \mathrm{um}$ electrodes could be pursued further or a new development method could be used to properly expose the electrodes with smaller diameters. This process would require a very precise aligning method of the mask, with minimal deviation from the negative photoresist mask. A different biocompatible negative photoresist could also be used which could be developed easily with using minimal development time. 


\section{Conclusion\Future Work:}

This thesis tested the applicability of using a MEA to measure impedance difference between melanoma and healthy skin cell. The results were inconclusive but do give an indication for future direction which could be useful in using MEAs to detect melanoma. The tests could be repeated by using methods outlined in the discussion section using tween to get rid of biological material present on the electrodes, in order to reduce the coating of organic matter on the electrodes. A methodology to get more precise readings would be to take impedance measurements over a range of frequencies. This would be useful because it would give characteristic impedance for the different types of tissues. The frequency vs. impedance waveform could be used to define each tissue separately and would allow for a more robust criterion for finding the two tissues. A cole-cole plot could allow for visually distinguishing the two types of tissues.

The future improvements outlined in the discussion and the conclusion section provide many suggestions to improve the signal to noise ratio such as the usage of the shunt resistor. The cleaning method presented in the discussion using tween could be used to remove organic material off the electrodes. Various other improvements outlined above would help distinguish tissue better and the improvements in wafer processing could be used to allow for electrodes to be used repeatedly. Using impedance spectroscopy to distinguish melanoma cancer cells from healthy skin cells can provide a cheap diagnostic method by employing materials and concepts prevalent in the semiconductor field. It could also aid in reducing cost of diagnosing melanoma by not having to do a painful biopsy or to do histology staining. This diagnostic method could 
also be used to detect melanoma cancer cells earlier so that the appropriate action can be taken before the cancer has a chance to spread to other parts of the body. 


\section{Appendix}

\section{General Linear Model: Impedance versus Frequency, Cook, Weight}

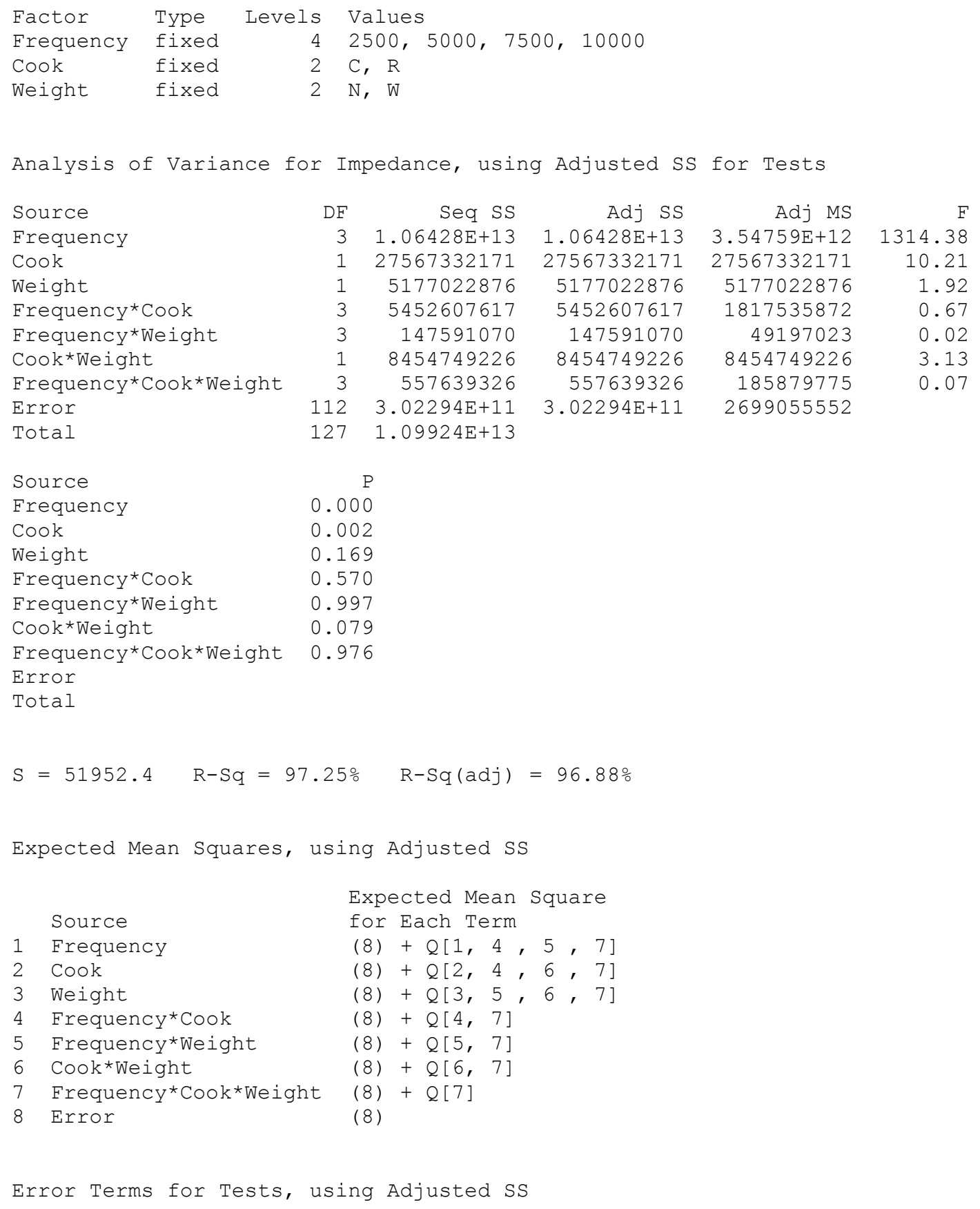


$\begin{array}{lllll}5 & \text { Frequency*Weight } & 112.00 & 2699055552 & \text { (8) } \\ 6 & \text { Cook*Weight } & 112.00 & 2699055552 & \text { (8) } \\ 7 & \text { Frequency*Cook*Weight } & 112.00 & 2699055552 & \text { (8) }\end{array}$

Variance Components, using Adjusted SS

$\begin{array}{lr}\text { Estimated } \\ \text { Source } & \text { Value } \\ \text { Error } & 2699055552\end{array}$

Figure 22. General Linear Model: Impedance versus Frequency, Cook, Weight

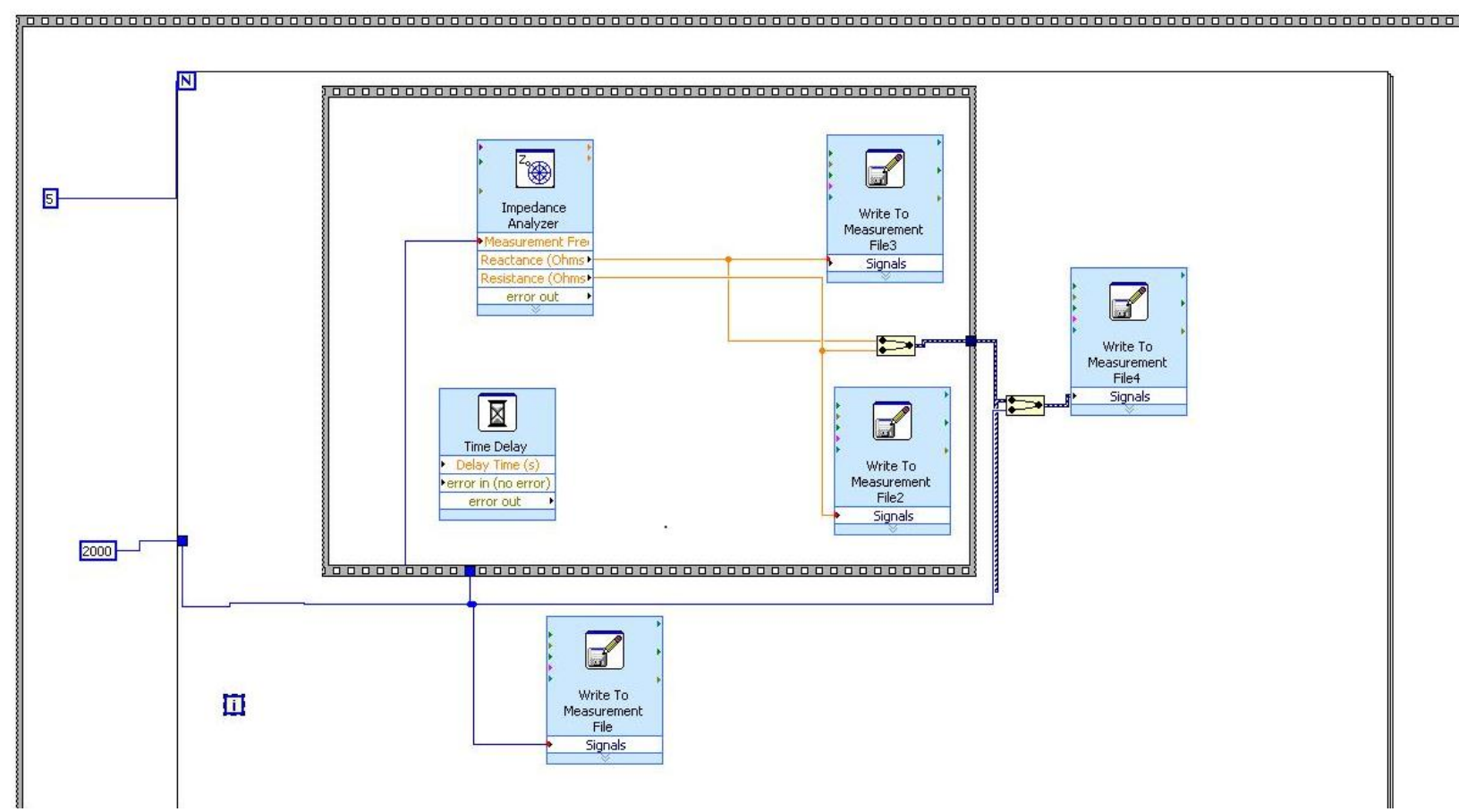

Figure 23. LabView code for Experiment III 


\section{References}

1. Fitzpatrick J, J.G.M., Dermatology Secrets in Color. Third ed. 2007, Philadelphia: Mosby Elsevier.

2. Friedman, J.R., Rigel, D.S., Kopf A., Early detection of Malignant Melanoma: The Role of

Physician Examination and Self-Examination of the Skin. Cancer Journal For Clinicians, 1985.

35(3): p. 21.

3. Melanoma Basics. 2010 January 2010 [cited 2012; Available from:

http://www.melanomacenter.org/basics/index.html.

4. Igarashi T., K.N., and Nayar S.K. The Appearance of Human Skin, in Computer Science. 2005, Columbia University: New York. p. 88.

5. What You Need To Know About Melanoma and Other Skin Cancers, N.C. Institute, Editor. 2010: Rockville. p. 59.

6. Aberg P., N.I., Ahansson J., Geladi P., Holmgren U., Ollmar S., Skin Cancer Identification Using Multifrequency Electrical Impedance --A Potential Screening Tool. IEEE Transaction on Biomedical Engineering, 2004. 51(12): p. 6.

7. Smoller, B.R., Histologic criteria for diagnosing primary cutaneous malignant melanoma. Modern Pathology, 2006. 19: p. 7.

8. Melanoma. Cancer Care Center 2011 [cited 2012 3/12/2012]; Available from: http://www.metrohealth.org/body.cfm?id=1636.

9. Macdonald, R., Impedance Spectroscopy. Annals of Biomedical Engineering, 1992. 20: p. 17.

10. Rahman A.R.A., P.D.T., Bhansali S., Effect of electrode geometry on the impedance evaluation of tissue and cell culture. Sensors and Actuators, 2007. B(127): p. 7.

11. Ivanic R., N.I., Rehacek V., Tvarozek V., Weis M., Thin film non-symmetric microelectrode array for impedance monitoring of human skin. Thin Solid Films, 2003. 433: p. 5.

12. Fleshman, S., Impedance Based detection of tissue using a multi-electrode device, in Biomedical and General Engineering. 2011, Cal Poly San Luis Obispo: San Luis Obispo.

13. Stante, G.C., The electrical properties of Human Tissue for the diagnosis and treatment of melanoma skin cancer, in Biomedical Engineering. 2009, California State Polytechnic University, San Luis Obispo: San Luis Obispo. p. 131.

14. McIntosh L.M., S.R., Jackson M., Mantsch H.H., Towards Non-Invasive Screening of Skin Lesion by Near-Infrared Spectroscopy. The Society for Investigative Dermatology, 2001. 116(1): p. 6.

15. Glickman, Y.A., Filo, O., David, M., Yayon, A., Topaz, M., Zamir, V., Ginzburg, A., Rozenman, D., Kenan, G., Electrical impedance scanning: a new approach to skin cancer diagnosis. Skin Research and Tehcnology, 2003. 9: p. 4.

16. Kress-Rogers, E., Handbook of biosensors and electronic noses Medicine, Food, and the Environment, ed. E. Kress-Rogers. 1997, New York: CRC Press. 4.

17. Zhang, Y., Zhang, X., Fang, J., Jiang, S., Zhang, Y., Gu, D., Nelson, R.D. Applications of SU-8 as the Insulator toward a Novel Planar Microelectrode Array for Extracellular Neural Recordings. in 2010 5th IEEE International Conference on Nano/Micro Engineered and Molecular Systems. 2010. Xiamen, China: IEEE.

18. Tijero M., G.G., Caro J. , SU-8 microprobe with microelectrodes for monitoring electrical impedance in living tissue. Biosensors and Bioelectronics, 2009. 24: p. 7.

19. Lorenz H, D.M., Fahrni N,LaBianca N ,Renaud P,Vettiger P SU-8: a low-cost resist for MEMS. Journal of Micromechanics and Microengineering, 1997. 7: p. 4.

20. Voskerician G., S.M., Shawgo R., Recum H., Anderson J.M., Cima M.J., Langer R., Biocompatibilty and biofouling of MEMS drug delivery devices. Biomaterials, 2003. 24: p. 8.

21. Melai J., C.S., Smits S., Visschers J., Schmitz J., The electrical conduction and dielectric strength of SU-8. Journal of Micromechanics and Microengineering, 2009. 19: p. 7. 
